\title{
An in vivo brain-bacteria interface: the developing brain as a key regulator of innate immunity
}

Celia Herrera-Rincon ${ }^{1}$, Jean-Francois Paré ${ }^{1}$, Christopher J. Martyniuk ${ }^{2}$, Sophia K. Jannetty ${ }^{1}$, Christina Harrison ${ }^{1}$, Alina Fischer ${ }^{1}$, Alexandre Dinis ${ }^{3}$, Vishal Keshari ${ }^{3}$, Richard Novak (D) $^{3}$ and Michael Levin (iD $)^{1,3 *}$

Infections have numerous effects on the brain. However, possible roles of the brain in protecting against infection, and the developmental origin and role of brain signaling in immune response, are largely unknown. We exploited a unique Xenopus embryonic model to reveal control of innate immune response to pathogenic $E$. coli by the developing brain. Using survival assays, morphological analysis of innate immune cells and apoptosis, and RNA-seq, we analyzed combinations of infection, brain removal, and tail-regenerative response. Without a brain, survival of embryos injected with bacteria decreased significantly. The protective effect of the developing brain was mediated by decrease of the infection-induced damage and of apoptosis, and increase of macrophage migration, as well as suppression of the transcriptional consequences of the infection, all of which decrease susceptibility to pathogen. Functional and pharmacological assays implicated dopamine signaling in the bacteria-brain-immune crosstalk. Our data establish a model that reveals the very early brain to be a central player in innate immunity, identify the developmental origins of brain-immune interactions, and suggest several targets for immune therapies.

npj Regenerative Medicine (2020)5:2; https://doi.org/10.1038/s41536-020-0087-2

\section{INTRODUCTION}

Innate immunity provides a first line of defense against pathogens ${ }^{1}$ and plays a crucial role in initiating adaptive immune responses. ${ }^{2}$ Most of the cell types of hematopoietic origin and genes known to be involved in mammalian innate and adaptive immunity have been identified in $X$. laevis, ${ }^{3,4}$ making the frog model an important emerging tool for understanding the cell and molecular biology of immunity. ${ }^{1,5,6}$ The immune response in the Xenopus embryo is provided uniquely by innate immunity during the first 12 days (d) post-fertilization, ${ }^{7}$ allowing studies of response to infection by wide range of pathogens without the confounding influences of adaptive immune component. ${ }^{5,8-11}$

An important emerging field concerns bidirectional intercommunication between two 'super-systems', the immune response and the brain, with implications for both basic evolutionary/cell biology and for biomedicine. ${ }^{12-14}$ Cytokines and other inflammation-related molecules affect vagal afferents or directly on brain, controlling aspects of behavior. ${ }^{15}$ Exciting recent studies integrating cognitive endpoints and cell biology of lymphatic vessels in the mature brain reveal the link between immune cells and brain functions. ${ }^{2,16-19}$ These studies reveal the adult brain as a regulator of adaptive immune response. ${ }^{20,21}$ The immune system has been proposed as a 'seventh sense', ${ }^{22}$ receiving information from pathogen agents to inform the central nervous system. Studies using invertebrate models suggest that neural circuits receive and integrate stimuli coming from pathogens, via $G$ protein-coupled receptors (GPCRs), to guide the immune response. . $^{23}$

Several key open questions remain in this fascinating field. First, while much work has been done on the role of the CNS in adaptive immunity, the interplay between brain and innate immunity is still poorly understood. Second, most of the data come from adult organisms, and the developmental origins of brain-immune interactions are largely mysterious. Last, an obvious limitation of mammalian models is the difficulty of performing loss-of-function studies that unequivocally show that the brain controls the immune system. ${ }^{26}$ To address these knowledge gaps, and to identify intervention strategies for innate immune function, we exploited a unique system for probing the interactions of brain and immunity in embryogenesis under normal conditions and when challenged with human pathogenic bacteria.

We developed a Xenopus model in which we could study braindependent events in embryogenesis: the brain is removed during early embryonic stages, but the animal can be kept alive and development continues. The ability of this vertebrate, a popular model for numerous biomedical contexts, ${ }^{27-34}$ to survive and develop without a brain provides a unique opportunity to understand the role of the brain in diverse systems-level outcomes. Our prior research into brain-dependent developmental signaling revealed that the nascent brain, even before being fully formed, plays an instructive role in patterning somitic muscle and peripheral neural networks. ${ }^{35,36}$ Here, we use this brainless vertebrate model, with intact spinal cord and peripheral innervation, to demonstrate an unknown role of the brain: regulating the early innate immunity in the presence or absence of infection. Our data, synthesizing a molecular comparison of infected and uninfected animals under normal, brainless, and tail-regenerative conditions, reveal the profound influence of the brain, in part mediated by dopamine signaling, upon susceptibility and response to pathogenic challenge at the cellular, molecular, and organism-wide levels.

\section{RESULTS}

Having a brain protects against infections

We previously showed that the uropathogenic $E$. coli UTI89 readily colonizes $X$. laevis embryos when infected at blastula or gastrula 
stages, ${ }^{9}$ and that survival rates at $4-5$ days after infection could be used as a readout of the degree of activation of the innate immune system. Here, we investigated the role of brain-derived signals in innate immunity and susceptibility by asking how animals respond to systemic infection in the absence of a brain (Fig. 1a).
Bacteria were injected into the blastocoel of embryos at $4.5 \times$ $10^{3} \pm 7.8 \times 10^{3} \mathrm{cfu} /$ embryo. First, we evaluated the survival rates at stage 48 (4-5 d after infection) for embryos subjected to the different infection conditions: infected with E. coli UTI89 (UTI condition) vs. not-infected (NI condition), belonging to each intervention or experimental group at st. 25 (Fig. 1b-e,
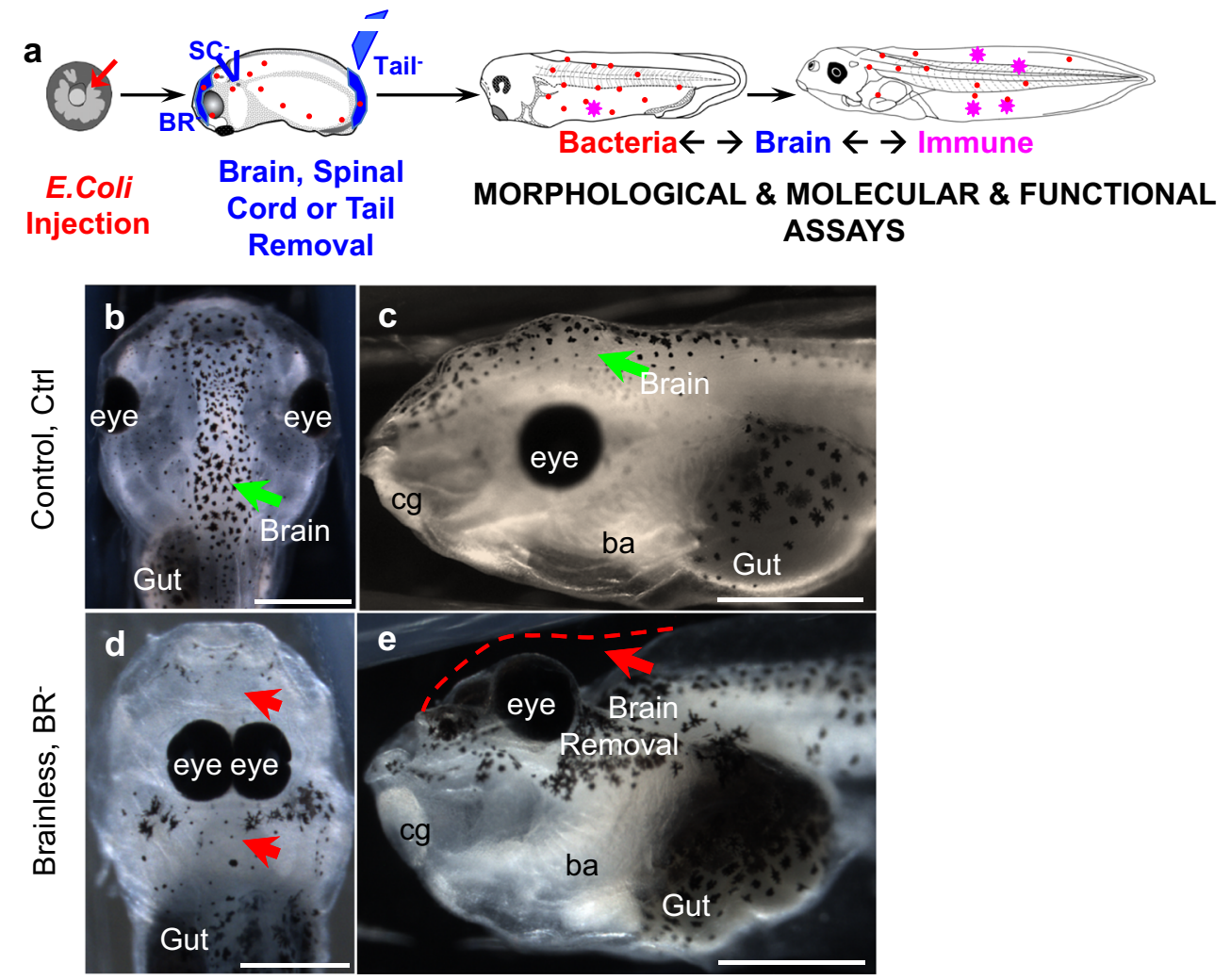

$\mathbf{f}$
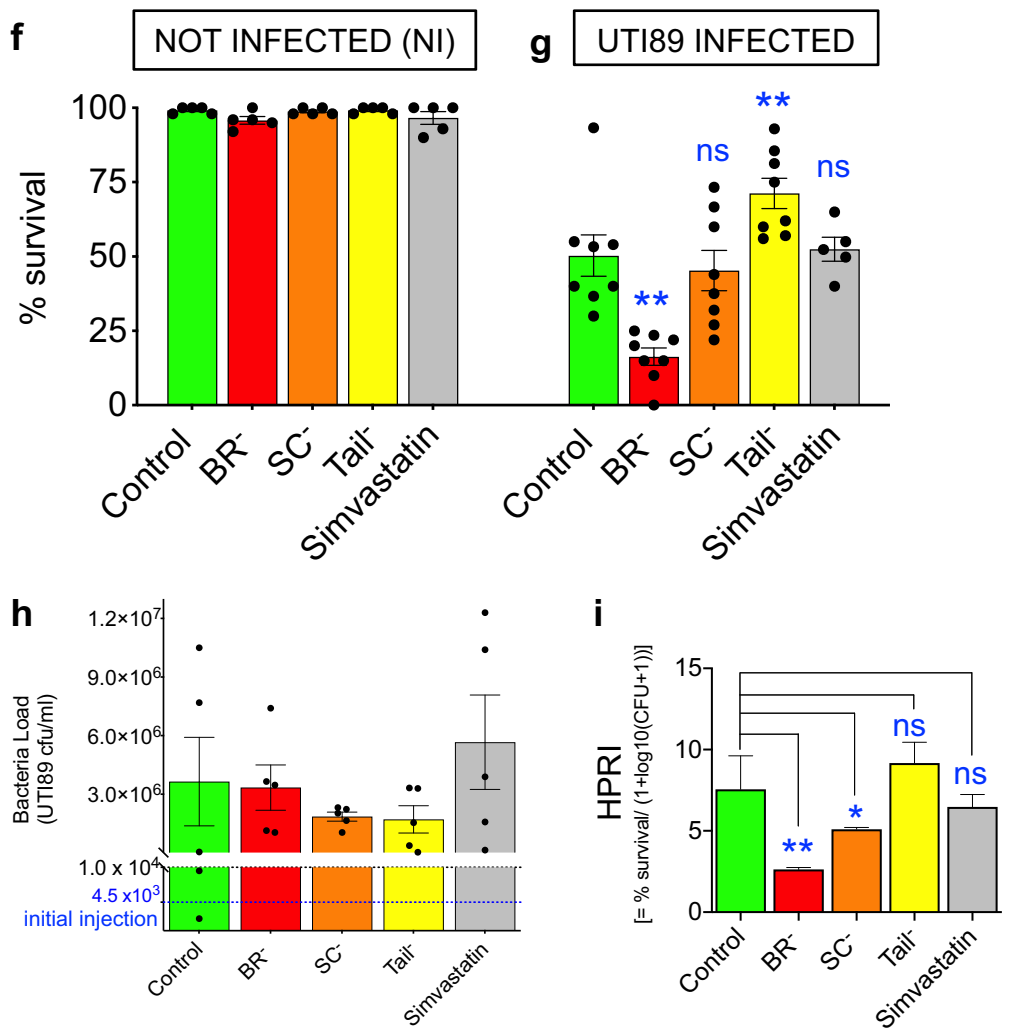
Fig. 1 The presence of a brain protects against systemic infection in Xenopus embryos. a One day after fertilization (early- to mid-gastrula or stage 12), Xenopus embryos were microinjected with the pathogenic bacteria E. coli UTI89. The next day, surgeries were performed for removal of the brain (brainless or BR ${ }^{-}$embryos), a piece of cervical spinal cord (SC embryos) or the tail bud (Tail embryos). Embryos were collected for morphological and molecular analysis during the next three days post-surgery. The bacterial load is represented in red. $\mathbf{b}$-e Dorsal (left column) and lateral (right column) views of st. 48 embryos belonging Control or Intact (Ctrl; b, c) vs. Brainless (BR ${ }^{-}$; d, e) experimental groups. Green and red arrows point, respectively, the control or correct vs. aberrant morphologies after surgery removal. Eyes, gut and branchial arches (ba) are indicated for reference. Left: rostral is up. Right: rostral is left, dorsal is up. Rostral is left and dorsal is up. Scale $\mathrm{bar}=500 \mu \mathrm{m}$. f, $\mathbf{g}$ Survival rates (plotted as percentage, \%) of each experimental group per each infection condition: without $E$. coli infection or not-infected animals (Nl; f, one-way ANOVA $P>0.05$ ) vs. E. coli UTI89 infected animals (UTI, evaluated four days after infection; g, one-way ANOVA $P<0.01$ ). Data represent the mean and S.D. of, at least, five independent replicates. Each replicate is shown by one dot. $\mathbf{h}$ Bacteria load measured and plotted as colony forming units per milliliter ( $\mathrm{cfu} / \mathrm{ml}$ ) in independent embryos (dots) belonging each experimental group or surgery condition. Alive embryos were harvested for analysis $48 \mathrm{~h}$ after infection. Initial bacteria load or number of bacteria injected (average of three independent replicates) at $t=0$ is plotted as a blue-dashed line. One-way ANOVA $P>0.05$. i Host-Pathogen Response Index (HPRI) $=$ $\%$ survival/ $(1+\log 10(C F U+1))$, for each experimental group, as a metric of tolerance. Data represent the mean and S.D. of five independent embryos. One-way ANOVA $P<0.01$. g, i $P$ values after post hoc Bonferroni comparisons are indicated as ${ }^{* *} P<0.01,{ }^{*} P<0.05$, ns $P>0.05$. See also Supplementary Fig. S1.

Supplementary Fig. S1): intact brain or control embryos (Control or Ctrl; Fig. 1b, c), brain removal (brainless or $\mathrm{BR}^{-}$; Fig. 1d, e; Supplementary Fig. S1a, b), resection of a piece of cervical spinal cord (SC ${ }^{-}$; Supplementary Fig. S1c, d), tail-bud amputation (Tailless or Taill $^{-}$), and muscle lysis via Simvastatin treatment (Simv; Supplementary Fig. S1h, j). At least, five biological replicates or dish of embryos ( $r=5, n=40$ embryos per replicate, $N$ total $=200$ embryos) were used per each group. Results were consistent among the replicates for each condition and group. For $\mathrm{NI}$ embryos, survival rates were similar among groups, ranging from 92 to $100 \%$ (99 $\pm 1 \%$ for Ctrl, $96 \pm 3 \%$ for $\mathrm{BR}^{-}, 99 \pm 1 \%$ for $\mathrm{SC}^{-}$, $99 \pm 1 \%$ for Tail ${ }^{-}$, and $97 \pm 5 \%$ for Simv; one-way ANOVA $P>0.05$; $r=5$ per group; Fig. 1f), suggesting any further differences among groups cannot be due to the surgical intervention itself. $E$. coli infection caused significant differences in the survival rates among groups (one-way ANOVA $P<0.01$; Fig. 1g), indicating its utility as infection-survival assay. For intact or Ctrl embryos the mean infection-survival rate was $50 \pm 20 \% \quad(r=8)$. Tail ${ }^{-}$animals expressed significantly higher survival rates with respect to Ctrl (71 $\pm 14 \%$, Bonferroni's posttest $P=0.018, r=8$ ), apparently resulting from the induced regenerative response. ${ }^{9,37}$ Conversely, infected animals developing without a brain $\left(\mathrm{BR}^{-}\right)$exhibited a low survival rate, with a significant drop to $16 \pm 8 \%$ (Bonferroni's posttest $P<0.0001, r=8$ ). The survival rate for $\mathrm{SC}^{-}$embryos (37 \pm $15 \%)$ was not significantly different than Ctrl group $(P>0.9999$, $r=8$ ), indicating that the effect was not due simply to surgical damage or the requirement for intact CNS overall. As a control for possible effects of general tissue distress on outcomes, and to ascertain potential impact of muscle structure on survival phenotypes, ${ }^{36}$ we tested Simvastatin treatment (Simv; Supplementary Fig. $\mathrm{S} 1 \mathrm{~h}-\mathrm{j})$. Simvastatin is a drug well-known to give severe collateral myotoxic effects in human patients ${ }^{38}$ and zebrafish embryos. ${ }^{39,40}$ It induces muscle disorganization similar to that observed after brain removal in Xenopus embryos, allowing independent characterization of the effects of brain removal vs. of muscle tissue lysis and disorganization. Simv group's survival was similar to that of Ctrl $(52 \pm 9 \% ; P>0.9999, r=5)$, demonstrating that the survival capacity and susceptibility to bacterial infection is not non-specifically modulated by a stressful combination of infection and strong tissue stressor.

We next wanted to ensure that the effects of the brain ablation were not due to the removal of migrating neural crest cells (NC; Supplementary Fig. S1e-g). Survival after NC ablation in absence of infection was similar to the other groups, $98 \pm 3 \%(r=3, n=40$, $N=120$ ), without any significant death related to the intervention itself. UTI infection in $\mathrm{NC}^{-}$embryos lead to a mean survival rate of $60 \pm 13 \%(r=3, n=40, N=120)$, similar to that reached in Ctrl animals (61 $\pm 30 \% ; \quad r=3, n=40, N=120)$ and significantly different from $\mathrm{BR}^{-}$group $(11 \pm 12 \% ; r=3, n=40, N=120$; oneway ANOVA $P<0.05$; Bonferroni's test for $\mathrm{NC}^{-}$vs. $C \operatorname{trl} P=0.9365$;
$\mathrm{NC}^{-}$vs. $\mathrm{BR}^{-} P=0.458$ ). Of the several tissues and organs we targeted, surgically and via lytic toxins, the brain was unique in its effects in promoting survival.

To further characterize the role of brain in modulating overall susceptibility to bacterial infection, we assessed the bacterial load in living embryos within each experimental group at $2 \mathrm{~d}$ after infection. Microbial analysis showed that the pathogen load was maintained among the different experimental groups, with an average of $3.24 \times 10^{6} \mathrm{cfu} / \mathrm{ml}$ (one-way ANOVA $P>0.05$; Fig. $1 \mathrm{~h}$ : the average initial dose of UTI E. coli injected was $4.5 \times 10^{3} \mathrm{cfu} / \mathrm{ml}$ ). Since the presence of brain improves survival but does not significantly reduce pathogen load, we analyzed our groups with an HPRI metric - an index that takes into account pathogen load as well as survival (Fig. 1i). Differences in HPRI index demonstrated that developing without an intact Central Nervous System (CNS; brain or spinal cord) significantly decreases tolerance ${ }^{41,42}$ and increases the susceptibility to infection (one-way ANOVA $P<0.01$ ).

Taken together, the results demonstrate the unique effects of brain removal on susceptibility to infection: survival depends on the presence of the brain regardless of any tissue injury, and the absence of brain makes embryos likely to succumb to bacterial infection.

Early brain protects against the infection-induced apoptosis

To understand the increased susceptibility to infection in $\mathrm{BR}^{-}$ animals, we conducted a longitudinal assay, scoring survival rates and characterizing apoptosis at several time points after infection and surgery ( $r=3, N=120$ per group; Fig. 2a, b). Embryos were analyzed at 2-4 h post-surgery (hps; corresponding to st. 26-27), 8 hps (st. 30), 24 hps (st. 35), 36 hps (st. 40), 48 hps (st. 42) and the end stage-48 time point ( 4 days ps). For all groups, the first wave of death was detected at $24 \mathrm{hps}$ (or $36 \mathrm{~h}$ post infection, hpi; Supplementary Table S1), specially for $\mathrm{BR}^{-}$animals, which went from $87 \pm 6 \%$ at st. 30 to $54 \pm 12 \%$ at st. 35 (two-way ANOVA $P<$ 0.01 ; Bonferroni's posttest $P=0.0005$ for $\mathrm{BR}^{-}$st. 25 vs. st. 35). For Ctrl animals, this peak (or first time point with significant differences respect to st. 25) was reached at st. 40 (48 hpi), with a drop in the survival rate to $68 \pm 23 \%$. At st. $40, \mathrm{BR}^{-}$animals showed a dramatic decreased survival rate $(19 \pm 7 \%)$, leading to significant differences with the rest of the groups, which kept constant until the end of the experiment (Bonferroni's posttest $P<0.01$ for $B^{-}$vs. C; see Supplementary Table S1 for over-time values at each stage and per each group). To characterize the cellular mechanisms behind the infection-induced death, we studied the patterning and number of apoptotic cells in Ctrl and $\mathrm{BR}^{-}$animals at the same longitudinal period (Fig. 2b). We used the antibody against Cleaved Caspase-3 (Asp175; CC3, which detects levels of activated caspase-3) as it has been extensively shown to be a reliable marker for apoptosis in Xenopus embryos. ${ }^{43-45}$ For both $\mathrm{Ctrl}$ and $\mathrm{BR}^{-}$groups, apoptosis followed a successive positive 

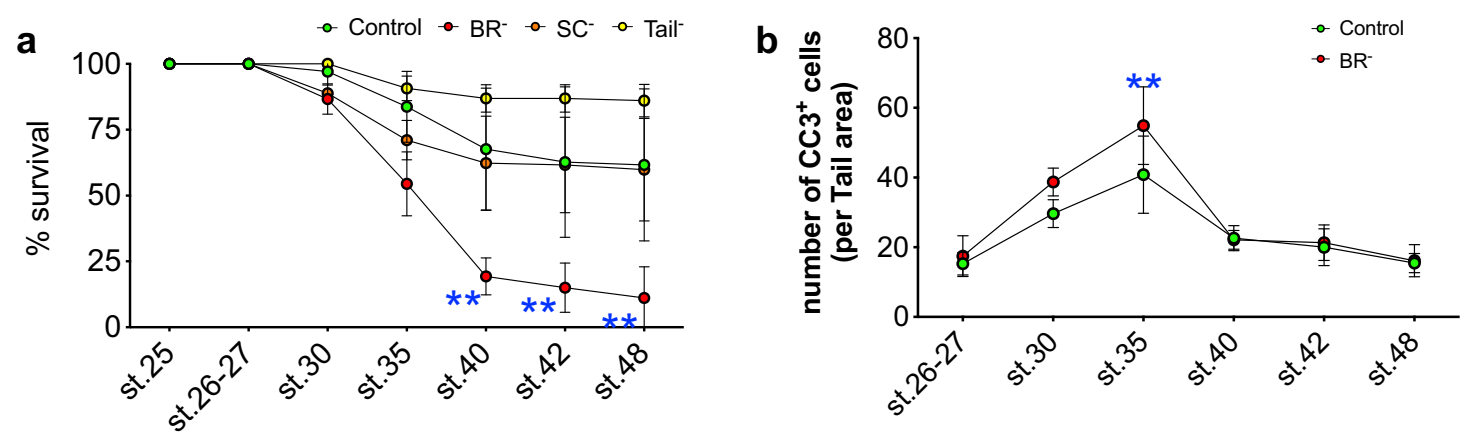

(2-4 hps)(8 hps)(24 hps)(36 hps)(48 hps)(4 dps)

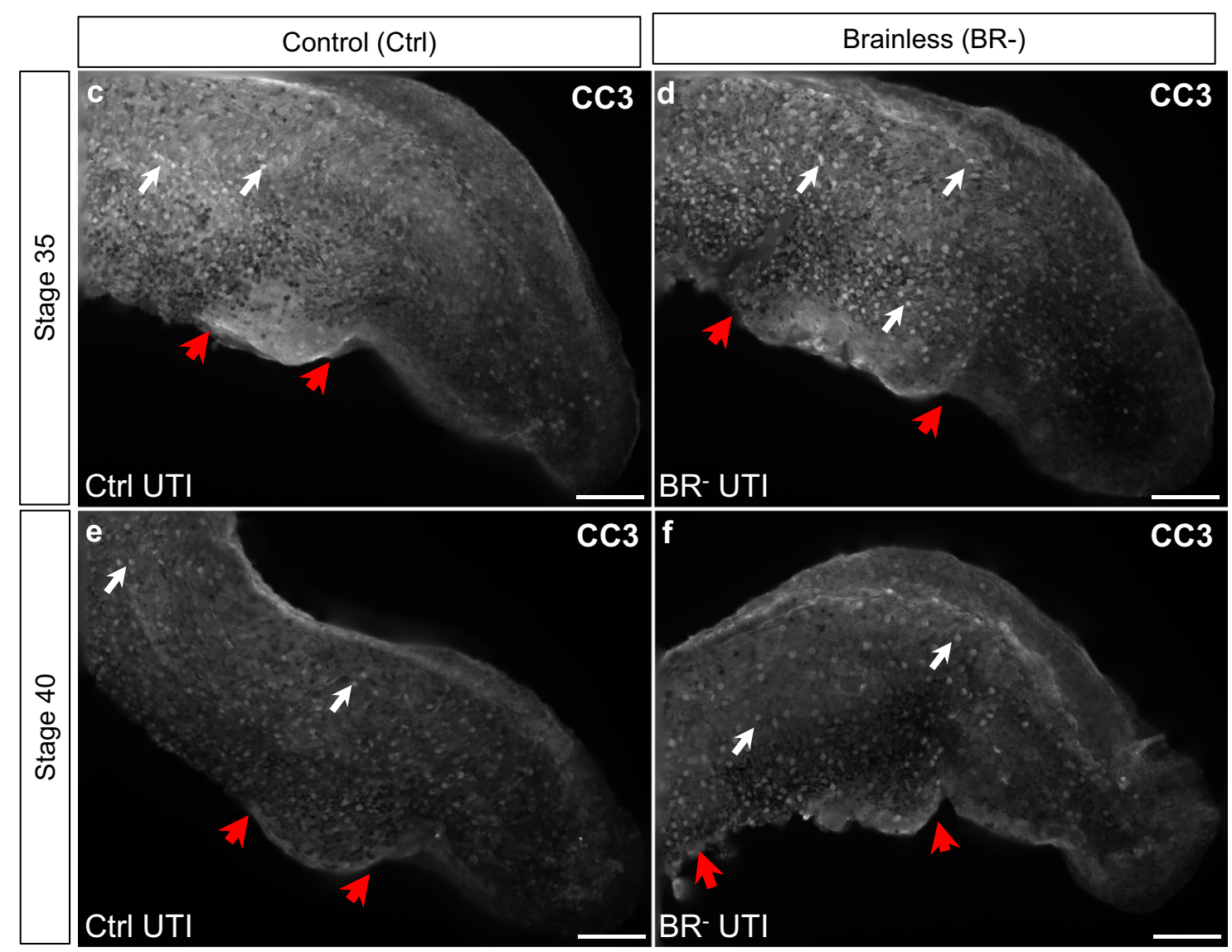

Fig. 2 The presence of a brain protects against infection-induced death, which is more severe during the first $48 \mathrm{~h}$ post infection. a Longitudinal assay, scoring survival rates after infection for Control (Ctrl, green), Brainless (BR ${ }^{-}$, red), Spinal Cord Resection (SC ${ }^{-}$, orange) and Tailless (Tail', yellow) embryos at 2-4 h post-surgery (hps; corresponding to st. 26-27), 8 hps (st. 30), $24 \mathrm{hps}$ (st. 35), $36 \mathrm{hps}$ (st. 40), $48 \mathrm{hps}$ (st. 42) and the end stage-48 time point ( 4 days ps). Data represent the mean and S.D. of three independent replicates. b-f Number (b) and expression pattern (c-f) of apoptotic cells (positively reacted against Cleaved Caspase-3 Asp175 antibody; CC3) in Ctrl (green) and BR (red) animals at the same longitudinal period than (a). Data represent the mean and S.D. of, at least, ten embryos coming from three different replicates. c-f Lateral views after CC3 immunofluorescence of UTI-infected Ctrl (c, e) and BR ${ }^{-}(\mathbf{d}, \mathbf{f})$ embryos at st. 35 (c, d) and st. 40 (e, f). White arrows point CC3-expressing cells; red arrows indicate damage related to the infection along the gut area. Rostral is left and dorsal is up. Scale bar $=250 \mu \mathrm{m}$. a, b Two-way ANOVA $P<0.01$. $P$ values after post hoc Bonferroni comparisons are indicated as ${ }^{* *} P<0.01$.

slope from st. $26-27$, reaching a peak at st. 35 , when significant differences are found in the number of cells between them $(41 \pm$ 11 vs. $55 \pm 11$ CC3-positive cells per area in Ctrl vs. BR ${ }^{-}$, respectively; two-way ANOVA $P<0.01$; Bonferroni's posttest $P=$ 0.0018; Fig. 2c, d). Morphological analysis of CC3 expression revealed that UTI Infection seems to affect mostly the gut region, characterized by a focus of highly reactivity to CC3 marker, which is focused on a posterior domain in Ctrl animals and occupying a more extensive area in $\mathrm{BR}^{-}$embryos (Fig. 2c, d). Animals at later stages in both groups expressed abnormalities in this region, but with decreased apoptosis, pointing this damage on the digestive domain as indicative of the UTI infection and defining the lethal consequences of infection to occur during the first $48 \mathrm{hpi}$. From st. 35 , apoptosis starts to decrease, being constant and without differences between groups until the end of the experiment (Fig. 2e, f). Taking together, these results show that death after infection-surgery occurs sequentially over the time, as a direct consequence of increased weakness, and it does not happen at the first immediate hours after surgery (st. 26-30). Interestingly, this analysis also shows that the peak of death in absence of brain 
occurs $24 \mathrm{~h}$ earlier than when the brain is present (at st. 35 vs. st. 40), with a significant increased apoptosis, supporting the protective effects of brain against the lethal consequences of the infection.

Brain signaling does not affect primitive myeloid precursors

Next, we asked whether the protective effects of the brain were due to action on the development of the primitive immune system (regardless of infection, as it does with muscle and nerve development) or whether, conversely, the brain acted on the active response to infection (implying differences between Control and $\mathrm{BR}^{-}$animals only after infection). In Xenopus, the earliest known marker of the primitive myeloid is spib-a (spiba). Spib- is a highly conserved ETS transcription factor that marks the primitive myeloid-cell lineage and is required for its development. $^{3,10}$ spib expression in myeloid precursors has been characterized in Xenopus, ${ }^{10}$ mouse, ${ }^{46}$ and humans. ${ }^{47}$ To specifically probe the role of the brain in establishing key cell types involved in innate immunity, we studied the spiba-positive ${ }^{10}$ cells in UTI and $\mathrm{NI}$ early-staged embryos (st. 28), subjected to the different surgical interventions (Ctrl, BR ${ }^{-}, \mathrm{SC}^{-}$, Tail'; Supplementary Fig. S2).

To compare the prevalence and spatial distribution of the spibapositive cell population, we characterized four independent regions: face, tail, dorsal flank, and ventral flank. At least, ten different embryos, from different replicates, per each group and condition were used for quantification $(r=3, n=40, N=120$; Supplementary Fig. S2a, b, t-w and Methods for details of quantification). NI embryos belonging to each experimental group possessed a similar number and distribution of positive cells, mainly located in the ventral region or hematopoietic organ at these stages (Supplementary Fig. S2c, d). In NI embryos, the only intervention that resulted in significant differences at the site injury from intact embryos was tail-bud amputation (one-way ANOVA $P<0.05$; Bonferroni's posttest $P=0.032$; Supplementary Fig. S2f). The number of spiba ${ }^{+}$cells in Tail ${ }^{-}$animals was especially higher in the posterior regions, occupying the bud and amputation plane. No differences from Ctrl embryos were detected for any area in $\mathrm{BR}^{-}$animals, suggesting the brain, in the absence of infection, is not necessary to maintain this population of primitive myeloid precursors.

Next, we analyzed the same cell population in embryos that were previously infected with E. coli UTI89 (UTI condition; Supplementary Fig. S2j-n). Neither intervention (brain, SC or tail removal) nor body region showed significant differences in number of spiba $^{+}$cells within infected embryos. Comparing effects of the infection on number of cells within each experimental group (Supplementary Fig. S2o-r), we detected a significant decrease in the total number of myeloid precursors as consequence of bacteria injection for all experimental conditions (two-way ANOVA $P<0.05$ ). This generic drop detected in st. 28 embryos indicates that infection with the human uropathogenic $E$. coli affects the primitive myeloid cells at early stages of immune system development. Intriguingly, tail amputation performed after infection, which showed the highest survival rates, did not induce a higher proliferation of spiba ${ }^{+}$cells, suggesting that the higher survival percentage observed in this group might not due to the presence of more myeloid progenitor cells.

Taken together, our results reveal the negative impact of infection at very early embryonic stages and demonstrate that the positive contribution of the brain to surviving infection is not mediated by brain-induced changes in the number of early myeloid-cell precursors.

Brain is required for migratory response of macrophages

Considering that the early brain does not control the primitive formation of myeloid cells at early stages, we decided to evaluate the dynamic behavior or migration of immune cells at later stages.
We labeled a gene coding for a protein expressed by Xenopus embryonic macrophages, $\mathrm{mmp}$, that mediates extracellular matrix remodeling, a crucial aspect of their migration. ${ }^{11,48}$ Homologs of this gene-protein have been extensively used to study macrophage behavior. ${ }^{10,11,48} \mathrm{mmp}$ 7-ISH was performed for the four experimental groups (Ctrl, $\mathrm{BR}^{-}, \mathrm{SC}^{-}$, Tail $\left.{ }^{-}\right)$per each infection condition (UTI vs. NI) in st. 36 embryos. At least, ten different embryos, from different replicates, per each group and condition were used for quantification $(r=3, n=40, N=120$; Fig. 3). In absence of infection ( $\mathrm{NI}$, Fig. $3 \mathrm{c}-\mathrm{g}$ ), the $m m p 7^{+}$population was only significantly different at the injury site for Tail ${ }^{-}$animals, as seen with the earlier myeloid spiba ${ }^{+}$cells, with an increased number of $\mathrm{mmp7}^{+}$cells accumulated in the bud tail or amputation plane respect to Ctrl embryos (one-way ANOVA $P<$ 0.01; Bonferroni's posttest $P<0.0001$; Fig. $3 \mathrm{~d}$ ). No differences were detected for any area between $\mathrm{Ctrl}$ and $\mathrm{BR}^{-}$animals, suggesting that the brain, in absence of infection, does not affect macrophage migration in the developing embryo. However, in embryos infected early in development with E. coli (UTI, Fig. 3h-I), absence of brain led to a significant increase in the number of $m m p 7^{+}$cells in ventral areas respect to Ctrl animals (one-way ANOVA $P<0.05$; Bonferroni's posttest $P=0.0464$ for $\mathrm{BR}^{-}$vs. Ctrl at ventral area; Fig. $3 \mathrm{k})$, represented by the accumulation of this population along the ventral hematopoietic niche (Fig. $3 \mathrm{~m}, \mathrm{n}$ ). The ventral clumping of mmp7-positive cells in $\mathrm{BR}^{-}$after infection was not detected in $\mathrm{SC}^{-}$ and Tail ${ }^{-}$embryos, resulting in significant differences between $\mathrm{BR}^{-}$ and Tail $^{-}$groups (Bonferroni's posttest $P=0.0225$ for BR ${ }^{-}$vs. Tail ${ }^{-}$ at ventral area; Fig. 3k), and demonstrating that the effect was not due simply to surgical damage or the need for an intact CNS.

The intragroup comparisons (Fig. 3o-r), analyzing embryos from the same surgery intervention but infected vs. not-infected conditions, and similarly to spiba ${ }^{+}$population, demonstrated that the overall number of $\mathrm{mmp}^{+}$cells was lower in infected embryos, regardless of experimental intervention. This result confirms that the human uropathogenic $E$. coli targets the early innate immune system. Interestingly, the differences previously detected between Ctrl and Tail ${ }^{-}$embryos in the tail area injury site, due to the tail injury itself, with a significant macrophage mobilization to the amputation plane (tail-bud area) observable only in absence of infection (Fig. 3d vs. Fig. 3i), were also displayed when comparing the effects of infection within the Tail ${ }^{-}$group (Fig. 3r). A significant difference in the number of macrophages at the tail region or amputation site is detected between not-infected and UTIinfected Tail ${ }^{-}$embryos (Bonferroni's posttest $P=0.0487$ for Tail $^{-}$ $\mathrm{NI}$ vs. Tail ${ }^{-}$UTI at tail region), indicating that the macrophage accumulation to the amputation plane in this group is entirely impaired in presence of bacterial infection. Our results show that the absence of the brain during development leads to defects in the response against infection, specifically the accumulation of $m m p 7^{+}$cells in the ventral niche reveals defects in macrophage migration.

Brainless induces ectopic myeloid and neural networks

Given the observed effects of the brain on the response of the developing innate immune ( st. 30), we next analyzed effects of the brain's presence on the fully-developed innate system ${ }^{5,7,49-51}$ and the surrounding innervation, in embryos at later stages of development ( $\sim$ st. 44-48; see Supplementary Note 1 for more details about embryogenesis of the immune system in Xenopus).

Immunofluorescence using the $\mathrm{XL}-2$ antibody ${ }^{8}$ revealed the distribution of all Xenopus leukocytes (monoclonal antibodies specific for particular leukocyte subpopulations are not currently available for these species). ${ }^{1}$ We quantified $\mathrm{XL}^{+}$cells along the middle and posterior parts of the animal body in not-infected and infected st. 44-45 $\mathrm{Ctrl}, \mathrm{BR}^{-}, \mathrm{SC}^{-}$, Tail ${ }^{-}$, and Simv embryos (Fig. $4 \mathrm{a}-\mathrm{h}$ ). In addition, we included the $\mathrm{NC}^{-}$group in the $\mathrm{XL2}$ analysis to study whether this intervention led to similar 
phenotypes of leukocyte migration than those ones expressed by $\mathrm{BR}^{-}$animals (Fig. 4e, f). At least, ten different embryos, from different replicates, per each group and condition were used for quantification $(r=3, n=40, N=120)$. Our results for $\mathrm{NI}$ animals showed similar leukocyte patterns and number among all the groups, being tail amputation the only intervention that provoked a differential increase of $\mathrm{XL}^{+}$cells in the tail region (two-way ANOVA $P<0.01$; Bonferroni's posttest $P<0.0001$ for Tail $^{-}$vs. the

$m m p 7^{+}$at stage $35-36$

a
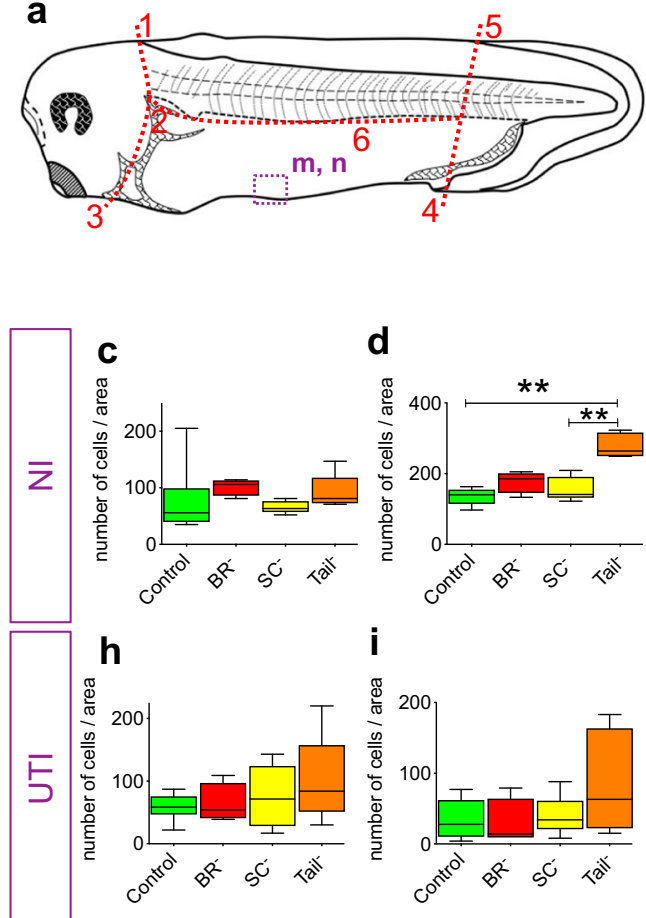

Face

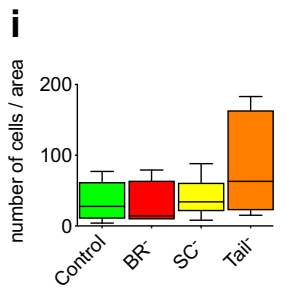

Tail e

b

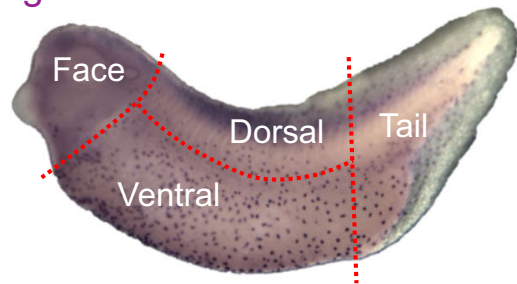
$\square$ Control $\square$ BR- $^{-} \square$ sC- $^{-} \square$ Tail $^{-}$
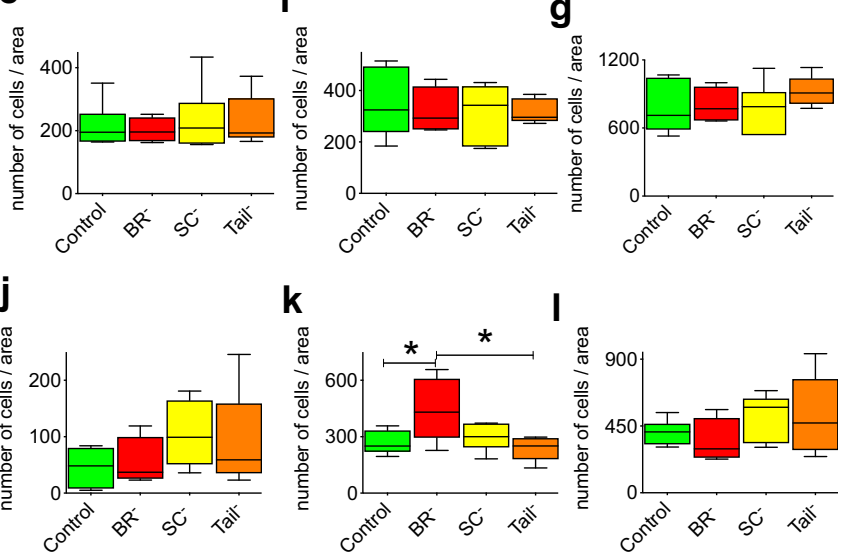

Dorsal

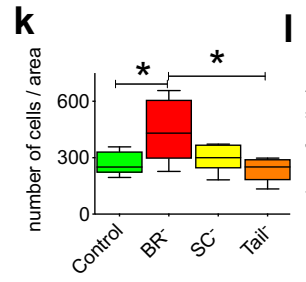

Ventral

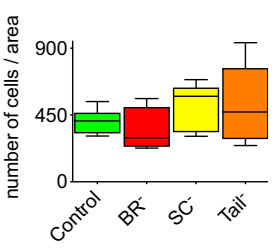

Total
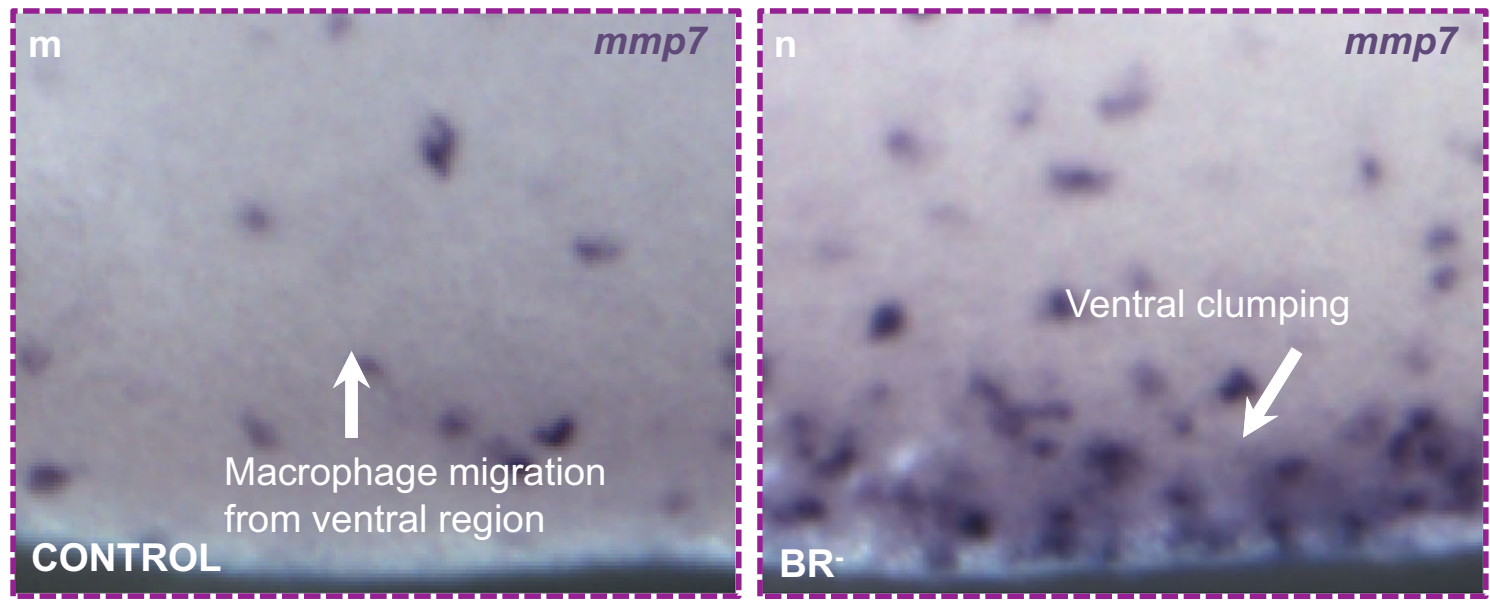

Not Infected (NI)

Infected (UTI)
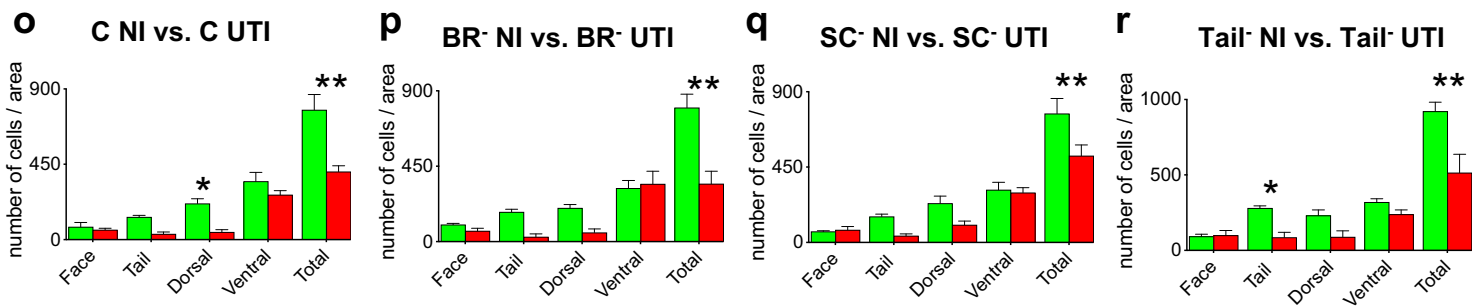
Fig. 3 The absence of the brain during development affects the migration of macrophages in response to infection. $\mathbf{a}$, $\mathbf{b}$ Drawing of (a) and image after in situ hybridization (ISH) for $\mathrm{mmp7}^{+}$(b) of one st. 35 Xenopus embryo indicating the landmarks or reference points (1-6) for the body subdivision in the four independent areas for quantification (see Methods for details). Dashed-purple line square indicates the area shown in $\mathrm{m}, \mathrm{n}$ inserts. Rostral is left and dorsal is up. c-I Quantification of number of $\mathrm{mmp} 7^{+}$cells in not-infected (NI, c-g) and infected (UTI, h-I), belonging each experimental group: Control (Ctrl, green), brainless (BR ${ }^{-}$; red), spinal cord resection (SC ${ }^{-}$; yellow) and Tailless (Tail ${ }^{-}$; orange). Values for normalized number of $m m p 7^{+}$cells are plotted per each region and group: face $(\mathbf{c}, \mathbf{h})$, tail $(\mathbf{d}, \mathbf{i})$, dorsal (e, j), and ventral (f, $\left.\mathbf{k}\right)$ to detect distribution and migration patterns. Total $(\mathbf{g}, \mathbf{I})$ is the sum of the four regions. One-way ANOVA $P$ value showed significance for (d) $(P<0.01)$ and $(\mathbf{k})(P<0.05)$. $\mathbf{m}, \mathbf{n}$ High-magnification images showing detail of ventral region (purple square in $(\mathbf{a}))$ with $\mathrm{mmp7-positive} \mathrm{cells} \mathrm{in} \mathrm{a}$ Control (m) vs. Brainless (n) embryos. VBI: Ventral Blood Islands or primitive hematopoietic organ. o-r Number of mmp7-positive myeloid cells (normalized to the area) in not-infected (green) vs. infected (red) embryos belonging Ctrl (o), BR ${ }^{-}(\mathbf{p})$, SC $\mathrm{SC}^{-}(\mathbf{q})$, and Tail ${ }^{-}(\mathbf{r})$ groups. Counts were done on four independent regions of the whole animal body (plus the summation): face, tail, dorsal and ventral areas. Two-way ANOVA showed significant $P$ values for all comparisons $(P<0.05)$. c-l, $\mathbf{0}-\mathbf{r}$ Data represent the mean and S.D. of, at least, ten different embryos, from three different replicates, per each group and condition. Significant $P$ values after post hoc Bonferroni test are indicated as $* P<0.05$ and ${ }^{*} P<$ 0.01. See also Supplementary Fig. S2.

rest of the groups; Fig. 4b). Then, we analyzed the XL2 expression in UTI-infected animals subjected to the different interventions. Remarkably, the population of XL2-positive cells after infection for all groups was 2-4-fold greater than in absence of infection, with leukocytes leaving the central blood vessels and spreading along the entire fin. UTI-infected $\mathrm{BR}^{-}$animals expressed the lowest $\mathrm{XL2}$ positive population ( $19 \pm 7$ vs. $49 \pm 10$ XL2-positive cells per area in $\mathrm{BR}^{-}$vs. Ctrl, respectively), with significant differences respect the rest of interventions (Bonferroni's posttest $P<0.0001$ for $\mathrm{BR}^{-}$vs. the rest of the groups).

Thus, these results confirmed our observations at early stages of development. While in absence of bacterial threat, brain is not required for specifying leukocyte number, in presence of bacterial threat brain is required to induce an appropriate responsemigrating behavior of the immune cells.

Next, we analyzed the transgenic xlurp::GFP animals, which express GFP in myeloid cells (mainly monocyte/macrophage and granulocyte/neutrophils at these stages) $)^{5,52}$ at st. $46-48$ of Ctrl, $\mathrm{BR}^{-}, \mathrm{SC}^{-}$, Tail ${ }^{-}$and Simv embryos. At least, ten different embryos, from different replicates, per each group and condition were used for quantification ( $r=3, n=40, N=120$; Fig. 4i-o, Supplementary Fig. S3, and Supplementary Video S1). One conspicuous feature observed in $\mathrm{BR}^{-}$animals was the presence of longitudinal-like patches of high-density of GFP-positive cells along the dorsal and ventral fin, not detected in the somite or central regions of the body. Consequently, we decided to evaluate the number of immune cells for each experimental group on two independent tail regions: center and periphery, with somite region and fin region, respectively (Fig. $4 \mathrm{i}-\mathrm{I})$. The central region after tail removal (Tail ${ }^{-}: 97 \pm 27 \mathrm{GFP}^{+}$cells) exhibited a marked increase in the number of myeloid cells (normalized to area) compared with Ctrl embryos ( $59 \pm 28 \mathrm{GFP}^{+}$cells; Dunn's posttest $P=0.0373$; Supplementary Fig. S3a, n), mainly focused at the region close to the amputation plane or injury site, indicating that even weeks after injury, myeloid cells are still invading the injured tail area. This effect was, conversely, not detected after brain removal, as more immune cells were not present either in the face region surrounding the injury site nor the central area. In the peripheral region, absence of the brain provoked a drastic increase in the number of myeloid cells at these late stages, forming defined patches that branched off the fin of the tail, at long distance from the injury site (from $30 \pm 21 \mathrm{GFP}^{+}$cells in Ctrl group to $86 \pm 15$ $\mathrm{GFP}^{+}$cells in BR ${ }^{-}$group; two-way ANOVA $P<0.05$; Bonferroni's posttest $P<0.0001$ ). This sprouted-network like patterning of the myeloid population in the fin region of $\mathrm{BR}^{-}$animals displayed a similar pattern to the one detected previously for the peripheral neural network in absence of brain. ${ }^{36}$

Thus, we decided to study the co-location of peripheral nerves with respect to this myeloid population (using Tub immunofluorescence on xlurp::GFP embryos). Morphological analysis revealed that, in central regions, myeloid cells of $\mathrm{BR}^{-}$exhibited different morphology, with respect to other areas or to Ctrl embryos, with rows of flattened cells, compatible with a macrophage-like network, ${ }^{5}$ following the internal neuropil. ${ }^{36}$ This distribution pattern, with myeloid cells in close proximity to the internal neuropil, was entirely absent in Ctrl embryos, where the central areas, with the highest number of GFP cells (Fig. 4i, j; Supplementary Fig. S3e-g) were not intensely occupied by nerves. In the peripheral region, a similar distribution pattern for both myeloid cells and peripheral nerves was detected in $\mathrm{BR}^{-}$, with scattered groups of immune cells in proximity to the highly disorganized neural networks (see the area circled within the dashed-white line as a illustrative example in Fig. 4m-0; Supplementary Fig. S3k-m for Ctrl). SC animals exhibited a similar number and general distribution of myeloid cells as the Ctrl group but the peripheral-nerve phenotype, as revealed by Tub immunofluorescence, showed the same ectopic growth and sprouting of neural network as in the $\mathrm{BR}^{-}$animals and significantly different from the Ctrl group (Supplementary Fig. S3o-q). Simvtreated embryos did not show differences for any marker, myeloid and nerve phenotypes, with respect to the Ctrl group.

Taken together, our results confirmed that brain removal during early development leads to the ectopic presence of mature myeloid cells at later stages of embryogenesis invading the fin, in close proximity to the aberrant peripheral neural network. This aberrant distribution of immune cells occurs far from the distal anterior injury site and is not mediated by spinal cord pathway. Lack of brain thus produces an immunologically different phenotype than other interventions, such as the removal of a different piece of the body (which induces the increased presence of immune cells but only at the local area of injury), or a severe toxic stress (Simv treatment, which caused no differences in myeloid cells relative to controls), indicating the unique influence of the embryonic brain on immune cell behavior.

\section{Brain controls the transcriptional consequences of infection}

Given the differences found for the innate immune response with or without a brain, we sought to identify the transcriptional mechanisms underlying the effects. To characterize the transcripts that could be differentially regulated under each condition, we conducted RNA-seq and compared the transcriptome of Ctrl and $\mathrm{BR}^{-}$embryos, with or without infection (Fig. 5, Supplementary Tables S1-S7, Supplementary Figs. S4 and S5, Supplementary Note 2, and Supplementary Data 1 and 2).

We first asked which transcripts are controlled by infection in an intact normal embryo by comparing the transcriptome of $\mathrm{NI}$ vs. UTI Control animals (Ctrl NI vs. Ctrl UTI, black labels in Fig. 5a). A total of 535 DEGs were regulated by infection in intact embryos, and 152 were unique to this group. Conversely, this number was significantly increased up to 719 (and 338 unique) transcripts controlled by infection in brainless embryos (or $\mathrm{BR}^{-} \mathrm{NI}$ vs. $\mathrm{BR}^{-}$ infected; orange labels in Fig. 5a), revealing that the presence of the brain suppresses the transcriptional consequences of infection 

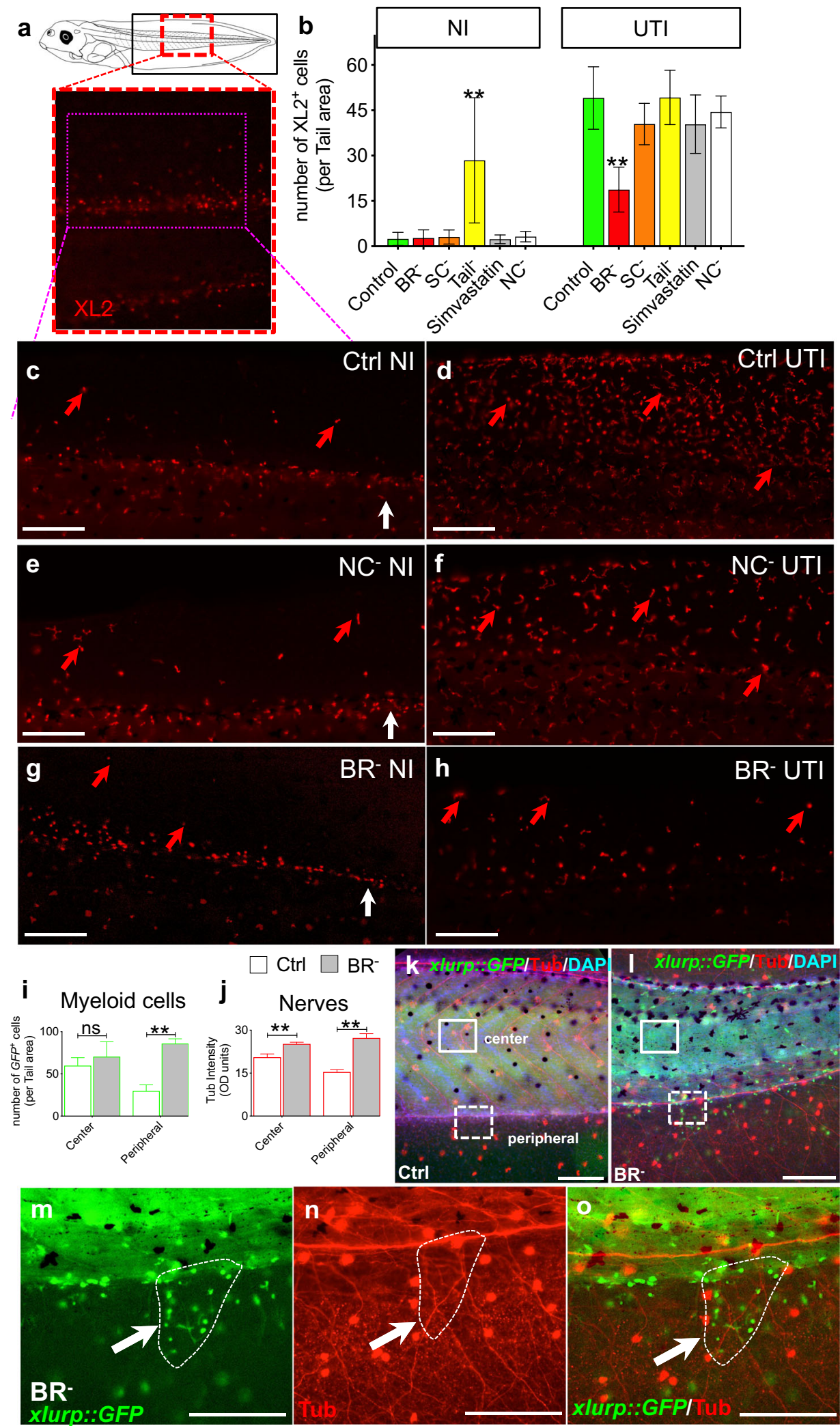

about twofold. In the absence of infection (comparing Ctrl NI vs. $\mathrm{BR}^{-} \mathrm{NI}$, blue labels in Fig. 5a), we found that 115 genes (and 37 unique) were responsive to brain removal per se. To characterize the transcriptional response specifically due to brain removal, and not to the removal of any organ in general, we compared the dataset of DEGs after brain removal from our study with the DEGs obtained after tail removal that were identified in two prior studies, in Xenopus ${ }^{53}$ and lizard. ${ }^{54}$ The tail is large appendage including a massive CNS component (spinal cord) and triggers a robust regenerative response, allowing us to identify and exclude 
Fig. 4 The absence of early brain affects number and patterning of myeloid cells, distributed in proximity to the aberrant neural network. a-h XL2 immunofluorescence to label leukocytes in uninfected (NI) and infected (UTI) embryos belonging Control (Ctrl, green), Brainless (BR ${ }^{-}$, red), Spinal Cord Resection $\left(\mathrm{SC}^{-}\right)$, Tailless (Tail ${ }^{-}$), Simvastatin-treated, and Neural Crest ablation (NC ${ }^{-}$) groups. a Drawing of a latestaged Xenopus embryo. Black rectangle indicates the tail area for counting. Magenta-dashed line outlines the region showed in (c)-(h). $\mathbf{b}$ Number of XL2 ${ }^{+}$cells (normalized to the area) per each group and condition. $\mathbf{c}-\mathbf{h}$ Lateral views of Ctrl $(\mathbf{c}, \mathbf{d}), \mathrm{NC}^{-}(\mathbf{e}, \mathbf{f})$, and BR $(\mathbf{g}, \mathbf{h}) ; \mathrm{NI}^{-}(\mathbf{c}, \mathbf{e}, \mathbf{g})$ vs. UTI (d, f, h) embryos. Red arrows point XL2 ${ }^{+}$cells. White arrows point the posterior region of the central blood vessels. $\mathbf{i}, \mathbf{j}$ Number of myeloid cells (i) and acetylated alpha-tubulin (Tub) optical density (OD; j) per each tail region for Ctrl (white), and BR (gray) st. 46-48 embryos. k-o Lateral views of $x$ lurp::GFP Ctrl (k) and BR ${ }^{-}$(I) embryos after Tub immunofluorescence. GFP ${ }^{+}$myeloid cells are green, Tub ${ }^{+}$nerves are red, nuclei are blue after DAPI staining. Differences in tail morphology for $\mathrm{BR}^{-}$animals (deviating the normal body axis (I) with respect to Ctrl (k)) are due to developmental muscle mispatterning occurring when brain is absent. Two independent tail regions were counted: center (occupied by the myotomes; solid-white line) and peripheral (dorsal and ventral lateral fins; dashed-white line). Counts were made at the same region of the animals (e.g., at the same somite level). $\mathbf{m}$-o Details from (I), white-dashed square. White arrows indicate positive elements at the same point on the three images. Dashed-white line circle an area within scattered groups of immune cells lying in proximity to the highly disorganized neural networks. b, i, j Data represent the mean and SD of, at least, ten different embryos, from different replicates, per each group and condition. Significant $P$ values after post hoc tests are indicated as ${ }^{*} P<0.01, \mathrm{~ns} P>0.05$. Images: rostral is left, dorsal is up. Scale bar $=250 \mu \mathrm{m}$. See also Supplementary Fig. S3.

from our analysis genes that are not specific to the removal of brain. After subtracting the common transcripts between brain and tail removal, we found that, from the initial 115 genes, 91 were unique to our dataset for brain removal and 24 were common with tail amputation (for the complete list of genes, see Supplementary Data 1, Brain Removal vs. Tail Removal). These data indicate that about $75 \%$ of the transcripts detected in our study after brain removal are unique to this intervention. The last comparison, Ctrl UTI vs. BR ${ }^{-}$UTI (green labels in Fig. 5a) revealed that the response to infection in the absence of brain affects the transcription of 218 (117 unique) genes.

Next, we extracted the specific genes with both the highest response (up- or downregulation) and unique (exclusively expressed) within each comparison (see Supplementary Data 1 for the complete list of genes; Supplementary Tables S2-S8 and Supplementary Note 2 for Summary of the top differentially expressed genes), concluding that most of the genes implicated in the bidirectional communication brain-infection belong to TNFR-I signaling pathway and encoding of ligands and receptors specific of immune/myeloid cells. Next, we grouped the differentially enriched elements according to their "large-scale functions" to reveal the types of processes mainly regulated within each brain-infection condition. The more predominant gene networks were related to blood, bone, ion transport or transmembrane potential (bioelectric signaling), ${ }^{55,56}$ apoptosis, development and regeneration, neural, immune response and bacteria (Supplementary Fig. S4a-d). The bioelectric-related genes (ion transport) occupied $3 \%$ of the total DEGs in control embryos under infection (Supplementary Fig. S4a), while this percentage was clearly lower in the rest of groups (around 1\%), consistent with the known role of bioelectric signaling in the innate immune response. ${ }^{9}$ The removal of the brain (in absence of infection) did not induce any changes in the transcription of genes related to bone $(0 \%)$, with the neural-related functions the most affected $(10 \%$ of the total of the transcriptome for $\mathrm{Ctrl} \mathrm{NI}$ vs. BR ${ }^{-} \mathrm{Nl}$; Supplementary Fig. S4c). How the absence of a brain affects the response to infection (Ctrl UTI vs. BR ${ }^{-}$UTI; Supplementary Fig. S4d) was reflected by considerable changes (respect to Ctrl NI vs. Ctrl UTI) in the percentages of immune-related transcripts $(-8 \%)$, neural-related $(+6 \%)$, bone $(-4 \%)$, and developmental and regeneration-related $(+2 \%)$ functions.

To reveal the gene regulatory networks (GRN) and motifs and features that were statistically overrepresented, we performed SNEA (Fig. 5c-f, Supplementary Figs S4 and S5, and Supplementary Data 1 and 2 for the complete list of pathways). In animals developed with a brain, 80 pathways were controlled by the infection (66 of them were unique to Ctrl NI vs. Ctrl UTI; black labels in Fig. 5b). Conversely, in animals developed without a brain (RNA isolation took place after brain removal), infection induced the differential regulation of 151 pathways (116 were unique to
$\mathrm{BR}^{-} \mathrm{NI}$ vs. $\mathrm{BR}^{-} \mathrm{UTl}$; orange labels in Fig. $5 \mathrm{~b}$ ), suggesting that about $70 \%$ of the response to infection depends on the presence of brain.

In grouping the neural-related pathways, we determined that three elements were shared by Ctrl UTI and BR ${ }^{-}$UTI (innervation, neurogenesis and brain microcirculation), five sub-networks were included exclusively in infected embryos with brain (Ctrl UTI: adrenergic transmission, CNS function, glia proliferation, brain blood flow and cerebrovascular circulation; Fig. 5c) and eleven were unique to infected embryos without brain $\left(\mathrm{BR}^{-}\right.$UTI: dopaminergic system, glial cell reaction, glial cell response, hindbrain development, hippocampus plasticity, nerve discharge, neural tube closure, neural tube patterning, neuron development, neuron differentiation, and peripheral-nerve function; Fig. $5 \mathrm{~d}$ ). Intriguingly, in the latter category, networks related to bacteria were upregulated by $20 \%$, overall as a group (Supplementary Fig. S4e). The only common pathways regulated in both response to brain removal and tail removal were microtubule- and cell division-related (cell cycle regulation, cell fate, chromatin remodeling, chromosome movement, epithelium development, microtubule bundling, microtubule cytoskeleton organization, microtubule sliding, and microtubule/kinetochore interaction). Some of the unique pathways differentially regulated after brain removal only, include apoptosis of neutrophils, apoptotic chromosome condensation, DNA annealing, DNA damage excision, DNA damage recognition, DNA end joining repair, DNA strand breakage, neurogenesis, neuron development, and neuron differentiation. The sub-network related to innate immune response was one of the most affected by the presence/absence of brain. Specifically, the innate immunity for brainless embryos with infection exhibited an upregulation of up to $11 \%$ (median change in the network of 1.11-fold; Fig. 5e). Some of these unique immune-pathways regulated in absence of brain were complement activation (classical pathway, Fig. 5f), immune complex clearance and activation, macrophage-focused (adhesion, apoptosis, fusion) and neutrophil-focused (activation, chemotaxis, extravasation and recruitment; all genes within a differentially expressed pathway are provided in Supplementary Data 2).

Thus, our analysis revealed profound changes in the transcriptional networks responsive to infection induced by absence of brain, suggesting a key role of the early brain in producing a robust innate immune response in response to systemic infection and implicating a variety of factors related to immune and neural pathways such as migration or dopaminergic transmission.

Dopamine is implied in the protective effects of the brain Considering the molecular candidates and pathways revealed by RNA-seq, we next targeted one of the regulatory networks exclusively affected after infection in the absence of the brain. From the 11 neural pathways unique to $\mathrm{BR}^{-} \mathrm{UTI}$, we decide to 
a

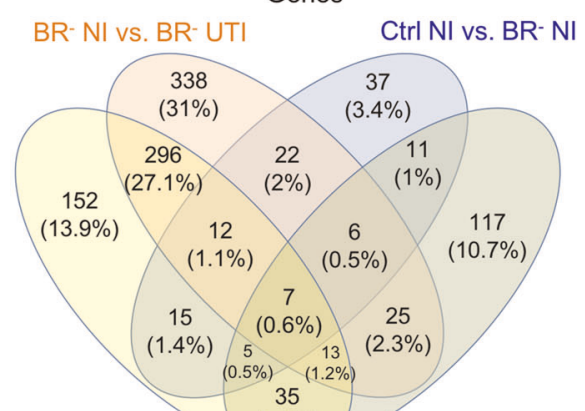

Ctrl NI vs. Ctrl UTI (3.2\%) Ctrl UTI vs. BR- UTI

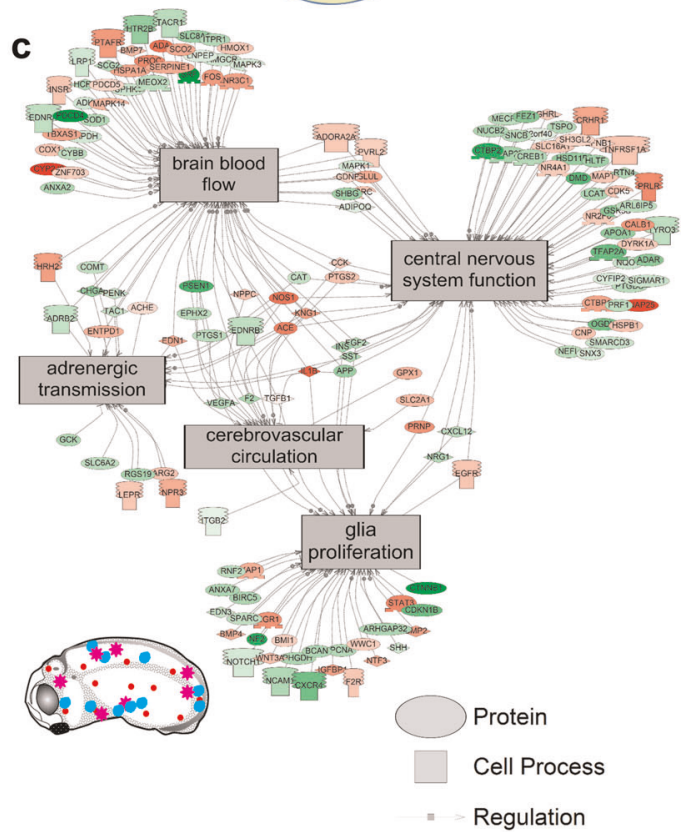

C

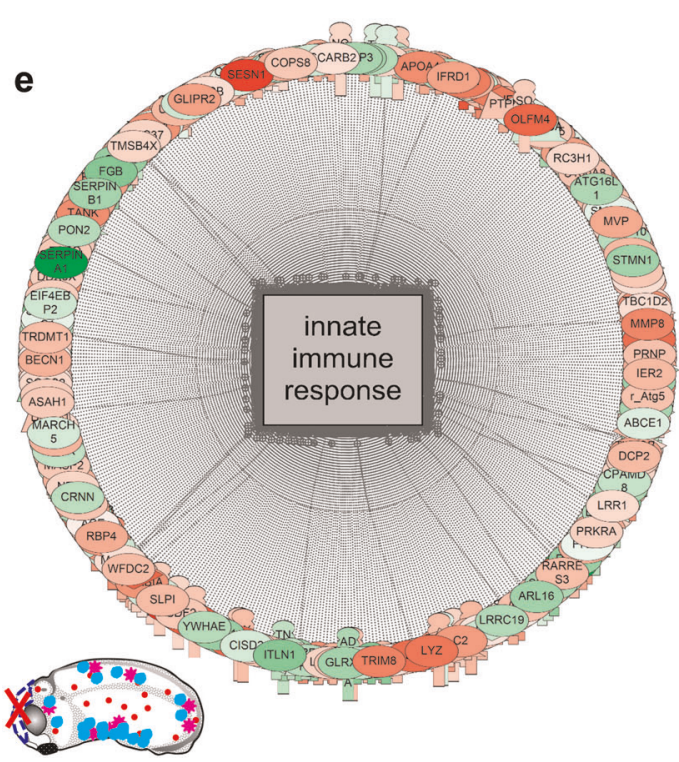

b

Sub-networks

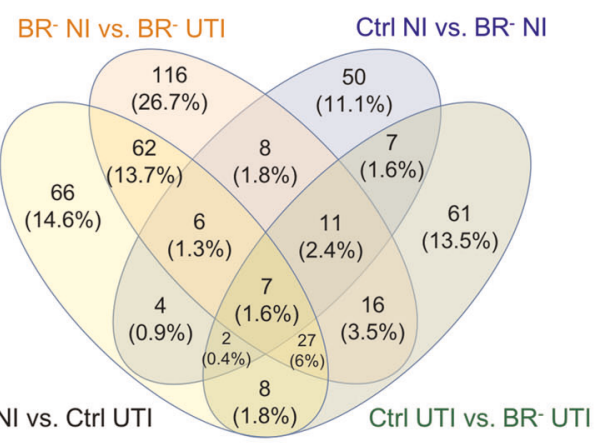

d
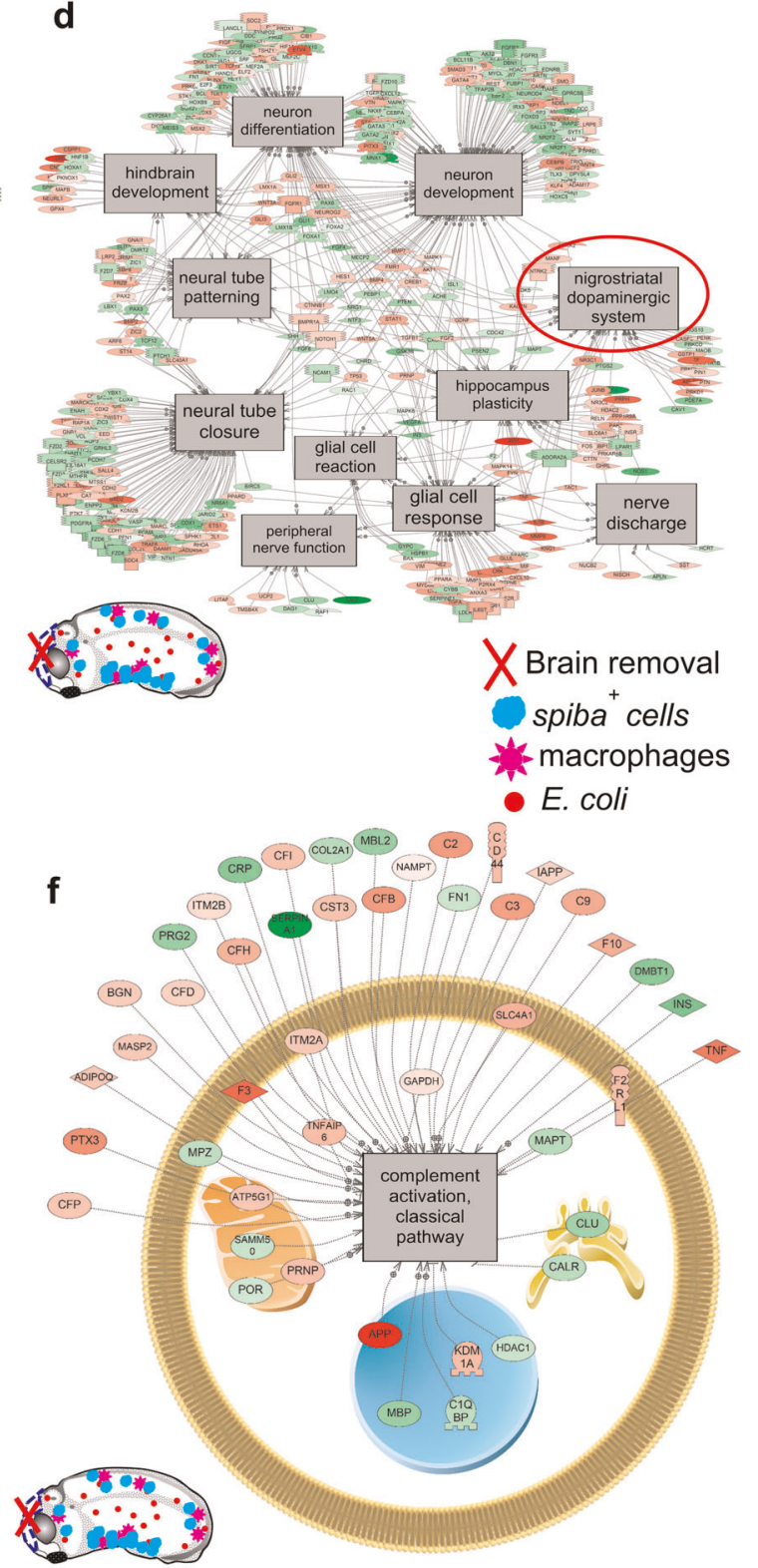

Fig. 5 Transcriptional analysis of Control (Ctrl) and Brainless $\left(B R^{-}\right)$datasets show quantitatively and qualitatively differences for regulated transcripts and cell processes after infection (UTI) or/and not-infection (NI) conditions. a, b Venn diagram comparing genes (a) and sub-networks (b) differentially regulated for each experimental group-condition. c Neural-related pathways unique to infection with a brain. d Neural-related pathways unique to infection without a brain. e Innate immunity response for brainless animals with infection. This network was significantly upregulated by $11 \%$. f Complement activation sub-network (classical pathway) exclusively present in infected embryos with absence of brain. Green = down gene, Red = up gene. Complete data are presented in Supplementary Data 1 . All measured genes found in a pathway are located in Supplementary Data 2. See also Supplementary Figs S4 and S5. 
validate the possible role of the dopaminergic signaling in mediating the increased susceptibility to infection, as it has been also implicated in macrophage migration. ${ }^{57}$

First, we functionally tested whether the DA levels were quantitatively different between $\mathrm{Ctrl}^{-}$and $\mathrm{BR}^{-}$infected embryos. Embryos were analyzed by LC-MS/MS at early st. 35 ( 20 h postsurgery, $44 \mathrm{~h}$ post infection), immediately before the peak of infection-induced death in $\mathrm{BR}^{-}$animals and the significant differences both in survival rate and apoptosis are reached respect to Ctrl embryos (Fig. 2a, b). DA turnover rates were significantly different between Ctrl $(35.56 \pm 10.49 \mathrm{pg} / \mathrm{uL})$ and $\mathrm{BR}^{-}$ $(14.90 \pm 0.99 \mathrm{pg} / \mu \mathrm{L})$ embryos $(r=3, n=30$; unpaired $t$-test $P=$ 0.0274; Fig. 6a, Supplementary Fig. S6), confirming that the DA concentration in the tail region of the embryo after infection is differentially affected in the presence vs. absence of the brain.

Next, we performed pharmacological assays targeting the type1 or type-2 family of dopamine (DA) receptors (D1R, D2R). We used specific agonists and antagonists of each dopamine receptor, ${ }^{58}$ such as the following: for D1R, ${ }^{57,59}$ SKF-38393 ${ }^{60}$ (SKF) and SCH$23390^{61}(\mathrm{SCH})$ were used as an agonist and antagonist, respectively; for D2R, Quinpirole ${ }^{57}$ (Quin) and L-741,626 ${ }^{62}$ (L741) were used as an agonist and antagonist, respectively. In order to understand whether we could mimic the $\mathrm{BR}^{-}$phenotype (decreased survival rate) in Ctrl embryos and/or mimic the Ctrl phenotype (increased survival rate) in $\mathrm{BR}^{-}$embryos, we exposed $\mathrm{Ctrl}$ and $\mathrm{BR}^{-}$infected embryos to the dopaminergic drugs from st. 25 (immediately after brain removal) to st. 48 , where we evaluated the survival rate after each treatment $(r=3, n=30, N=90$ embryos per treatment; Fig. 6b). For Ctrl infected embryos, no significant differences in the survival rates were found after exposure to any of the drugs. For $\mathrm{BR}^{-}$infected embryos, exposure to $\mathrm{SCH}$ (D1R antagonist) rescued embryos from the increased death $(14.8 \pm 8 \%$ with no drug) to a survival rate of $36 \pm 6 \%$, displaying significant differences respect to the values reached for the rest of no-drug/drug-treated $\mathrm{BR}^{-}$embryos (two-way ANOVA $P<0.01$; Bonferroni's posttest for $\mathrm{BR}^{-}$: $\mathrm{SCH}$ vs. $\mathrm{MMR} P=0.0049$; $\mathrm{SCH}$ vs. SKF $P=0.0015$; $\mathrm{SCH}$ vs. Quin $P=0.0006$; $\mathrm{SCH}$ vs. L741 $P=$ 0.0071 ) and no differences respect to no-drug Ctrl embryos (48 \pm $7 \%$, Bonferroni's posttest $P=0.1878$ for $\mathrm{BR}^{-} \mathrm{SCH}$ vs. Ctrl. MMR). These results confirmed, using functional assays, that the dopaminergic regulatory network is affected in $\mathrm{BR}^{-}$infected embryos, and that antagonism of the D1R signaling pathway can rescue the induced-infection death detected in absence of brain, mimicking the protective effects of the brain signals on the immune response to infection.

\section{DISCUSSION}

Immunology, developmental biology, neuroscience, and regenerative medicine are all converging in an emerging interdisciplinary field of profound significance for biomedicine as well as basic biology. ${ }^{63}$ While the brain-immune axis is now beginning to be characterized, ${ }^{9,17,64}$ many gaps exist in our understanding of the functional links between the brain, response to infection, and regenerative processes triggered by wound healing. ${ }^{65}$ This is especially true for the earliest developmental events that shape the future interactions of these subsystems during health and disease. Here, we exploited the highly tractable Xenopus laevis model to identify unrevealed aspects of the innate immune response that rely on the presence of brain, and we characterized the cell-level and transcriptional machinery that underlies the effects.

We challenged Xenopus embryos with UTI E. coli load of $4.5 \times$ $10^{3} \mathrm{cfu} / \mathrm{ml}$; this dose is up to five orders of magnitude smaller than some model organisms and human organs tolerate, ${ }^{66-69}$ providing a comfortable dynamic range within which to evaluate susceptibility and tolerance. Crucially, we show that the specific presence of the brain strongly impacts the ability of embryos to survive bacterial challenge (Fig. 1). The early brain is protecting the embryo by inducing cellular and physiological responses (decreasing the infection-induced damage and apoptosis; Fig. 2, and promoting macrophage migration; Figs 3 and 4) and molecular mechanisms (suppressing transcriptional consequences of the infection; Fig. 5) that help to overcome the bacterial threat. Pharmacological and functional assays revealed that absence of brain during infection leads to decreased levels of peripheral DA. Targeting the DA-D1R signaling pathway in brainless animals rescues them from infection-induced death (Fig. 6). These results suggest that brain-derived DA signaling is key in mediating the protective effects of the brain signals in response to infection.

The brain is not necessary for the early development and proliferation of primitive myeloid precursors (Supplementary Fig. S2). However, in response to infection, signals from the brain are required for proper macrophage migration (Fig. 3), revealing that brain-dependent signals regulate the behavior of immune cells. Without infection, at later stages with a more mature immune system, the absence of a brain leads to aberrant and ectopic distribution of macrophages and neutrophils (Fig. 4), which occurs in the context of an ectopic neural network which could be a factor guiding the abnormal distribution of immune cells (Fig. $4 m-0)$. Importantly, removing the brain does not induce the migration of immune cells to the injury site (the head region), which clearly occurs with other parts removed from the body, such as tail amputation (Supplementary Fig. S3n).

The protective effects of the brain do not require spinal cord contiguity, an important fact for the design of immune-enhancing therapies. Survival rate of $\mathrm{SC}^{-}$embryos after infection is similar to Ctrl (Fig. 1i), and mature immune cells in $\mathrm{SC}^{-}$animals have similar numbers and general distribution as the control group (Supplementary Fig. S3a, b). Conversely, the peripheral-nerve phenotype in $\mathrm{SC}^{-}$animals showed the same ectopic growth and sprouting of neural network than in $\mathrm{BR}^{-}$animals (Supplementary Fig. S3o-q). Thus, unlike the peripheral-nerve distribution, immune cell behavior is not controlled via the spinal cord. Likewise, neural crest ablation did not recapitulate effects of brain removal (Supplementary Fig. S1e-g), consistent with the essential role of the brain per se.

Important information was provided by two additional groups: tailless (a control for general surgical damage), and the Simvastatin-treated group (Simv, a control for general tissue stress). Simvastatin causes severe myotoxic effects in humans and zebrafish embryos, ${ }^{39,40}$ leading to death from continuous exposure or above $\mu \mathrm{M}$ concentration, indicative of a strong drugderived stress. In Xenopus embryos, Simv treatment led to severe aberrant muscle phenotypes, without altering the brain morphology (Supplementary Fig. S1h-j). Survival after infection (Fig. 1f) and the immune phenotype (Supplementary Fig. S3) of the Simv group are not significantly different than the Ctrl group, indicating that muscle alterations are not a primary cause of immune defects, and the combination of infection + strong stressor does not reduce survival. Thus, the absence of brain when a bacterial infection is present leads to dysfunctional macrophage behavior and high susceptibility to infection that is not recapitulated by even severe general stressors or tissue damage.

To more fully understand the link between brain and immunity, we assessed the bacterial load at $48 \mathrm{~h}$ after infection and the relation between survival and pathogen load, using a metric that combines these two variables in a single number: Host-Pathogen Response Index (HPRl; Fig. 1i). Comparing survival percentage across the control infected vs. brainless infected vs. brainless noninfected (Fig. 1f, g) under constant pathogen load confirmed brain-dependent susceptibility, which includes a tolerance component (Fig. 1h, i). In addition to the brain, it is likely that other components of the circuit could be discovered in the future. The cellular and morphological mechanisms behind the higher susceptibility in absence of the brain include increased apoptosis 

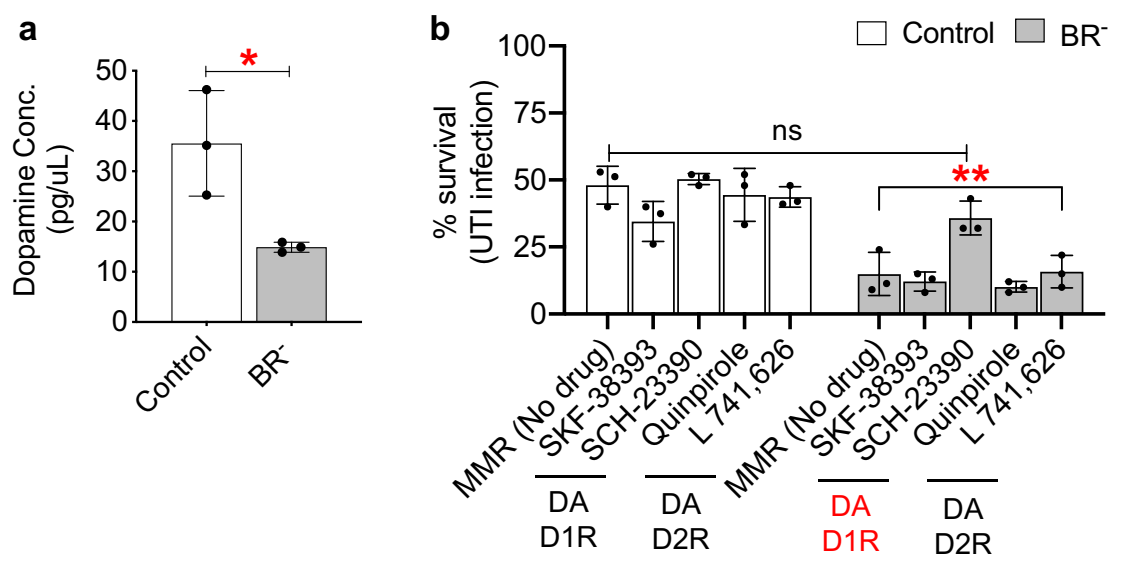

c Presence of infection, in presence of brain, leads to :

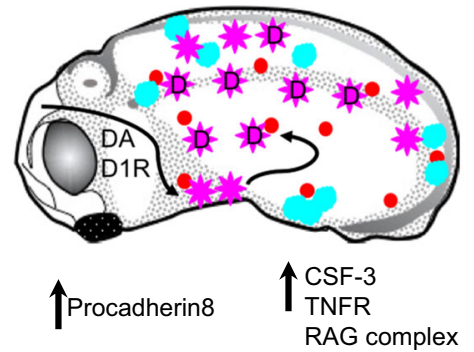

I Neutrophil elastase $\checkmark$ Actin-filament network

Adrenergic transmission Glia proliferation

$\begin{array}{ll}\text { Protein } & \text { X Brain removal } \\ \text { Cell Process } & \begin{array}{l}\text { DA-activated } \\ \text { Regulation }\end{array} \\ \text { Unique Transcripts } & \text { Apoptotic cells } \\ \text { Unique sub-networks } & \text { E. coli }\end{array}$

d Absence of brain, in presence of infection, leads to :

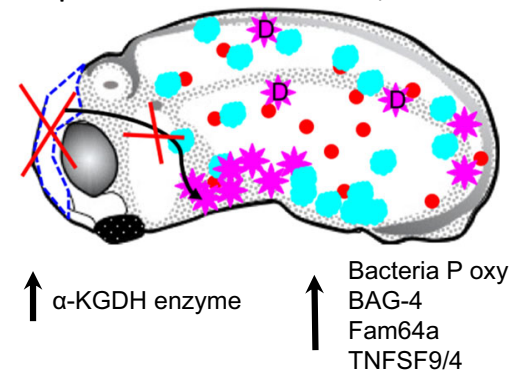

Dopaminergic transmission Glial reaction

Peripheral nerve function

Complement activation

Immune complex clearance

Macrophage adhesion, apoptosis \& fusion

Neutrophil activation, chemotaxis, extravasation

Cbl

mRNA uridylation

Axin-2

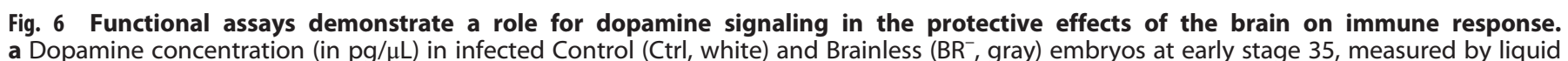

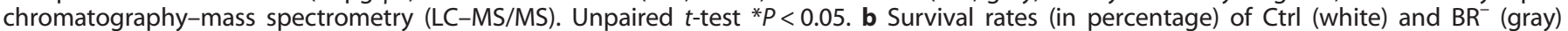

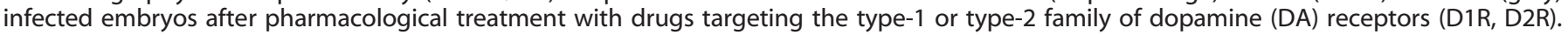

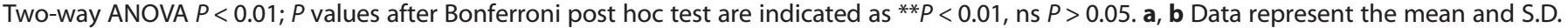

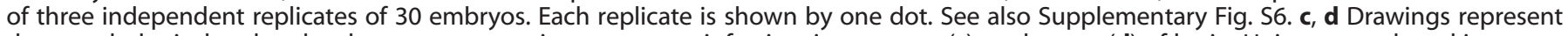

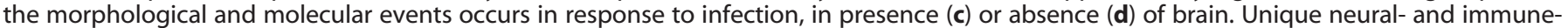

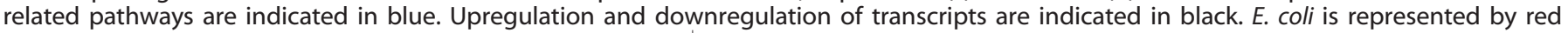

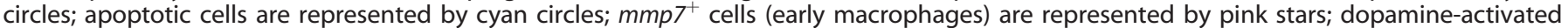

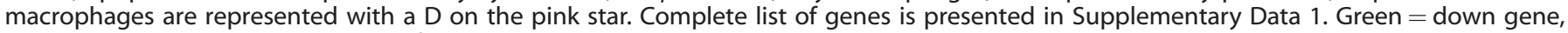
Red = up gene. All measured genes found in a pathway are located in Supplementary Data 2.

and inflammation induced by infection (Fig. 2), which promote an earlier peak of death and hamper the recovery from infection and normal development to st. 48.

Analysis of the embryos' robust transcriptional response shed light on the pathways related to brain $\rightarrow$ body, bacteria $\rightarrow$ body, and bacteria $\rightarrow$ brainless body signaling. Infection when brain is present induces the differential expression of immune-related elements such as antigen recognition, leukocyte cell adhesion, lymphoid differentiation, T-cell proliferative response and tolerance, basophil activation, disease resistance, thrombocyte aggregation, and virus morphogenesis. Infection. Similarly, some of the more significantly upregulated genes, include genes encoding proteins (ligand-receptor) for immune cell functioning (Fig. 5, Supplementary Fig. S5), such as the gene encoding colonystimulating factor 3 (CSF 3, a granulocyte growth factor necessary for the differentiation of bone marrow cells to granulocytelineage ${ }^{70}$ ), the gene encoding for receptor-interacting serine/ threonine-protein kinase 3-like (RIP, a component of the Tumor Necrosis Factor-receptor I (TNF-R1) signaling complex, ${ }^{71}$ and RAG complex genes involved in maturation of the antibody repertoire of adaptive immunity. The differential regulation of the TNFsignaling pathway is consistent with recent mammalian data 
showing that innate immune response in mice to Listeria monocytogenes results in upregulation of TNF in cerebrovascular fluid. ${ }^{2}$

The deficient response to infection detected in brainless animals, with low survival rates and defects in immune cell location, was transcriptionally reflected, with an increased activity of the innate immune sub-network by $\sim 11-12 \%$ (Fig. 5e) compared with animals with a brain. The ineffective upregulation of transcripts may indicate compensatory transcriptional responses in the body from other systems in the absence of a brain when challenged with a pathogen. ${ }^{72}$ RNA-seq revealed the most significant immune and neural pathways and transcripts affected by absence of brain such as complement activation, macrophage-focused (adhesion, apoptosis, fusion), neutrophilfocused (activation, chemotaxis, extravasation and recruitment), or overexpression of BAG-4 related genes (or TNF-R1 silencers) and cell adhesion proteins (VCAM-1). Networks functionally related to bacteria are inhibited by $20 \%$ and eleven neural pathways are uniquely affected after infection when brain is not present.

The dopaminergic transmission is one of the most affected regulatory networks in presence of infection when the brain is absent, and levels of peripheral DA are decreased in absence of brain. Macrophage migration is one of the targets for the protective role of the brain and an increasing number of recent studies relate dopamine and inflammation and immunity. ${ }^{57,73,74}$ Based on our cellular, molecular and pharmacological results, a possible mechanism explaining the protective effects of the brain in presence of bacteria is illustrated in the drawings-models of Fig. $6 \mathrm{c}$, d. In presence of bacteria, the immune response of the embryo is initiated in the early brain (st. 25) and communicated to the periphery by modulating DA-signaling pathways in macrophages (trough D1R antagonism) during the first $48 \mathrm{~h}$ post infection (or before st. 35). The DA-activated macrophages migrate and act systemically decreasing apoptosis and infection-induced inflammation. As a consequence, embryo's tolerance to infection is harnessed and by st. 40, induced-infection death is entirely stabilized. In absence of brain, low levels of peripheral DA cannot activate immune cells and promote them to initiate the migratory response. Consequently, in absence of a macrophage network, brainless embryos become more susceptible to the lethal effects of the bacterial infection, which induces high levels of inflammation and apoptotic events that lead to an earlier and massive peak of death by st. 35 .

Brainless animals showed an aberrant overexpression of central(nigrostriatal dopamine) and peripheral- (peripheral-nerve function) neural networks (Fig. 5d), as well as bacteria-related pathways (Fig. S4e), revealing responses of the host to microbial presence that could explain the differences in the HPRI and, consequently, the lower survival rates detected in brainless animals after infection. Specifically, the TNF-R1 pathway is significantly upregulated in intact embryos (developed with brain) in presence of infection, and it seems to be deregulated when brain is absent. Brainless infected animals overexpress genes related to BAG-4 or silencer of death domains (SODD) - a widely expressed 60-kiloDalton protein that associate with the death domain of TNF-R1, silencing it when overexpression is detected. ${ }^{75}$ The accumulation of immune cells in niche in absence of brain (Fig. $3 m, n$ ) is also detected in transcriptome changes, with the significant upregulation of the vascular cell adhesion protein 1 (VCAM-1) that mediates the adhesion of immune cells. Cell cycle and chromatin-expression regulators, such as ubiquitination of proteins (mediated by tnip1 or cbl), methylation (via downregulation of euchromatic histone-lysine $\mathrm{N}$-methyltransferase $1 \mathrm{~L}$ that methylates the lysine- 9 position of histone $\mathrm{H} 3$ and tags it for transcriptional repression) or uridylation of mRNAs appear significantly and exclusively regulated in brainless animals (with or without infection). In infected animals, absence of brain induces the specific networks for ubiquitization of CSF-1R (macrophage receptor for growth and proliferation). This mechanism, along with high expression of adhesion proteins, could be the responsible for the attenuation in the macrophage proliferation detected in brainless animals.

In conclusion, a unique vertebrate model that develops without a brain enabled morphological, transcriptional and functional evidence for brain-mediated modulation of the immune system reactivity. Specifically, we demonstrate that the absence of brain makes embryos more susceptible to pathogen, lowering tolerance ${ }^{41,42}$ and increasing apoptosis and inflammation. The influence of the endogenous brain seems to be mediated by control of cell localization, especially affecting macrophage migration to fight infection. RNA-seq revealed the most significant immune and neural pathways and transcripts affected by absence of brain. Overall, $70 \%$ of the response at the cellular network level is different based on the presence/absence of brain. Our functional assays reveal that DA signaling could be key in the bacteria-brain-immune crosstalk, suggesting that modulation of dopamine receptors could be an important strategy for mimicking the protective effects of brain signals on the immune response to infection in biomedical settings.

The brain is an active component of the innate immune response. Future work will focus on decoding the bioelectric and biochemical signals that mediate its effects on distant immune cells and identifying heretofore unrecognized cellular targets of these signals. More broadly, beyond the brain-body-bacteria axis, the understanding of the influence of the brain over cell- and molecular-level processes is an interesting frontier, with numerous potential applications across basic biology and medicine. A full understanding of brain effects on cellular behavior (complementing neuroscientists' focus on whole animal behaviors) is likely to not only shed light on the evolution of neural and immune systems but also to facilitate the development of intervention strategies in biomedical settings. We speculate that combinations of appropriate bioelectrical and neurotransmitter signals can become a useful tool for addressing infectious and other disease states.

\section{METHODS}

Animal husbandry

Xenopus laevis wild-type and transgenic embryos were fertilized in vitro according to standard protocols ${ }^{76}$ in $0.1 \mathrm{X}$ Marc's Modified Ringer's solution (MMR; $10 \mathrm{mM} \mathrm{Na}^{+}, 0.2 \mathrm{mM} \mathrm{K}^{+}, 10.5 \mathrm{mM} \mathrm{Cl}^{-}, 0.2 \mathrm{mM} \mathrm{Ca}{ }^{2+}, \mathrm{pH}$ 7.8). The transgenic Xenopus, expressing Green Fluorescent Protein (GFP) under the control of the lurp1 gene promoter, xlurp::GFP, was obtained from the Marine Biological Laboratory (MBL, National Xenopus Resource RRID: SCR_013731)..$^{77}$ Xenopus embryos were housed at $18-21^{\circ} \mathrm{C}$ and staged according to Nieuwkoop and Faber. ${ }^{78}$ All experimental procedures involving Xenopus embryos were approved by the Institutional Animal Care and Use Committees and Tufts University Department of Laboratory Animal Medicine under protocol M2017-53.

Bacterial injections, survival and host-pathogen assays

All bacterial strains were generously provided by Dr. Matthew A. Mulvey (University of Utah, Salt Lake City, Utah, USA) and have been previously described. ${ }^{79}$ We utilized an uropathogenic Escherichia coli (E. coli) strain UTI89 to elicit a pathogenic response. Bacteria were grown at $37^{\circ} \mathrm{C}$ in LB medium (MP Biomedicals, LLC) supplemented with $50 \mathrm{mg} / \mathrm{mL}$ ampicillin (Fisher). One milliliter of the UTI89 stock $\left(1 \times 10^{9}\right.$ colony forming units per $\mathrm{ml}(\mathrm{cfu} / \mathrm{ml})$ ) overnight bacterial culture was pelleted and resuspended to a stock concentration of $1 \times 10^{10} \mathrm{cfu} / \mathrm{mL}$ in phosphate-buffered saline before injections in embryos. Gastrula stage embryos were injected using borosilicate glass needles calibrated for a bubble pressure of 25-30 kDa and $150 \mathrm{~ms}$ pulses. All known variables were kept consistent across biological and technical replicates. To calculate the injected cfu, embryos from different biological replicates were harvested immediately after injection $(t=0 \mathrm{~h})$ for subsequent lysis and plating. Later, embryos infected with E. coli UTI 89 (UTI condition) and not-infected (NI condition) were incubated at $21^{\circ} \mathrm{C}$ (Fig. 1a for general diagram of experimental design). 
Surgeries were performed $18-24 \mathrm{~h}$ after infection (st. 25). Survival within each experimental group was evaluated at several stages until embryos reached st. $46-48$, or $4-5 \mathrm{~d}$ after infection. Even though bacteria were grown and concentrated following a constant methodology, and embryos were collected and grown under the same conditions, variation in survival rates was observed between experiments. This is likely due to differences in bacterial growth in situ and in the genetic background of each individual from different egg clutches. For these reasons, every individual comparison within an experiment was conducted using eggs from a single fertilization and a single suspension of bacteria, and controls were used within each study. At least 120 embryos were used for every treatment being compared, and each assay was tested in triplicate. Three additional injection assays were performed in order to calculate the number of bacteria per embryo at $48 \mathrm{~h}$ after infection. Bacterial load was determined after lysis of live embryos (five independent embryos/experimental group; TissueLyser for $30 \mathrm{~s}$ at $30 \mathrm{~Hz}$ ) and following standard protocols of microbial plating analysis. Timing selection was established following previous published work, ${ }^{9}$ which demonstrated that the lethal effect of UTI89 infection on Xenopus embryos can be evaluated at $72 \mathrm{~h}$ and the survival rates maintain constant after $5 \mathrm{~d}$. The Host-Pathogen Response Index $(\mathrm{HPRI}=\%$ survival $/(1+\log 10(\mathrm{CFU}+1))$ was developed to compare embryo survival with respect to pathogen load in order to provide a quantitative metric of susceptibility and tolerance that comprises both pathogen load and survival. HPRI scores of 0 indicating complete susceptibility and 100 indicating complete embryo survival and clearance of pathogen. The slope of HPRI over time or following a perturbation, reflects tolerance phenotypes.

\section{Experimental groups and microsurgery}

At 18-24 h post infection, st. 25 infected (UTI condition) and not-infected ( $\mathrm{NI}$ condition) embryos were randomly allocated in one of the four experimental groups, respectively (Fig. 1b-e, Supplementary Fig. S1): Control (Ctrl; Fig. 1b, c), Brainless (BR ; Fig. 1d, e), Spinal Cord resection (SC ; Supplementary Fig. S1c, d) or Tail-bud resection (Tail ${ }^{-}$). The $\mathrm{SC}^{-}$group was used to test whether the effects are mediated by neural communication going through the spinal cord. We used Tailless embryos as control for invasiveness of removal of large amounts of tissue and as a positive control, since we have previously demonstrated this assay increases resistance to infection. ${ }^{9}$ Brain removal was performed under dissecting microscope, using a dissecting knife (FST \#10055-12), via a single cut of the antero-dorsal region corresponding to the three main subdivisons of the developmental brain (see Supplementary Fig. S1a, b for images of stage-48 embryos showing that no brain regeneration takes place after removal at st. 25). For $\mathrm{SC}^{-}$, a consistent piece of the most cervical levels, sizing 50-100 $\mu \mathrm{m}$ in length, was completely removed by using two forceps with super-fine tips (Dumont \#5ST, FST 11252-00). Tail buds were amputated from the most posterior fifth portion of their body using a scalpel blade (additional details of microsurgeries can be found in ref. ${ }^{36}$ ). As an additional experimental control for the brain ablation, we included the group Neural Crest ablation ( $\mathrm{NC}^{-}$; Supplementary Fig. S1e-g). Published protocols for ablation of the migrating cranial NC in Xenopus ${ }^{80}$ were followed at st. 17 embryos, $\sim 6-8 \mathrm{~h}$ after infection.

After surgery, animals were allowed to heal in $0.75 \mathrm{X}$ MMR for $1 \mathrm{~h}$, then raised in regular $0.1 \mathrm{XMMR}$ at $21{ }^{\circ} \mathrm{C}$ and scored and analyzed for percentage of survival rate at st. $26-27$ (2-4 hps), st. 30 ( $8 \mathrm{hps})$, st. 35 ( $24 \mathrm{hps})$, st. 40 ( $36 \mathrm{hps})$, st. 42 ( $48 \mathrm{hps})$, and st. $46-48$ (4 days ps). At each time point, embryos were harvested and prepared for morphological analysis (in situ hybridization or immunofluorescence). Molecular assays, RNA extraction and liquid chromatography-coupled tandem mass spectrometry (LC-MS/MS), were performed on whole embryos harvested at 3 and 20 hps, respectively.

Stage-25 UTI and NI Xenopus embryos were treated with $0.4 \mu \mathrm{M}$ Simvastatin (Supplementary Fig. S1h-j; Tocris Cat. No. 1965/50) for $18 \mathrm{~h}$ at $22^{\circ} \mathrm{C}$. After 18 -h exposure, embryos were transferred to fresh normal media (0.1X MMR) and allowed to develop up to the same stages as the rest of the groups for harvesting and posterior analysis. Stock solution of the drug was created by dissolving the compound in ethanol to a final concentration of $0.24 \mathrm{mM}$. Further dilution was made in 0.1X MMR.

\section{In situ hybridization and cell counting}

In situ hybridization was performed as previously described ${ }^{9}$ on UTI and NI embryos belonging each experimental group: $\mathrm{Ctrl} \mathrm{BR}^{-}, \mathrm{SC}^{-}$, and Tail' Briefly, specimens were washed in $0.1 \%$ Tween-20/phosphate-buffered saline (PBS-T) and dehydrated through increasing concentrations of methanol. Xenopus laevis spib-a (spiba; GE Dharmacon) and Xenopus tropicalis $\mathrm{mmp} 7$ (matrix metalloproteinase-7) were used as probes and kindly provided by Enrique Amaya (University of Manchester). spib- is an ETS transcription factor, highly conserved with mammals, ${ }^{10,47,81}$ necessary for myeloid specification. spiba expression at st. 28 embryos marks the earliest primitive myeloid population. ${ }^{3,10} \mathrm{mmp} 7$ is a secreted metalloproteinase involved in extracellular matrix remodeling and extensively used as macrophage differentiation and migration markers., ${ }^{3,10,11,48,82} \mathrm{mmp} 7$ probe was used to label migrating embryonic macrophages on st. 35-36 embryos, as relative to other myeloid markers, $\mathrm{mmp7}$ is expressed considerably later. $^{10}$ Probes were generated in vitro from linearized templates using a digoxigenin (DIG)-labeling mix (Sigma-Aldrich, \#11277065910 Roche).

Quantification of spiba- (Supplementary Fig. S2) and mmp7- (Fig. 3) positive cells was performed using ImageJ software on bright-field images of whole-mount ISH st. 28 and st. 35 embryos, respectively. Images and counts were performed on the left side of the animals belonging each experimental group ( $\mathrm{Ctrl}^{\mathrm{B}} \mathrm{BR}^{-}, \mathrm{SC}^{-}, \mathrm{Tail}^{-}$) and for each condition (NI vs. UTI). Firstly, each image was carefully subdivided and cropped (to avoid overlapping) in four independent (face, tail, dorsal and ventral) using six reference points or landmarks (Fig. 3a, b, Supplementary Fig. S2a, b, s-w). Clearly identifiable landmarks for Xenopus embryos were orderly used (from 1 to 6 ) to decrease variability and bias during the blind counting, such as the following: (1) beginning of spinal cord (SC), most anterior portion or cervical levels, (2) intersection between posterior edge of the IV branchial arch (ba) and ventral edge for the first and most anterior somite (Sm); (3) anterior to the heart (h); (4) end of hindgut (hg); (5) beginning of the tail bud (tb); (6) ventral line delimited by somites. The face region is composed of the entire anterior area including the olfactory bulb, the eye area, and the otic vesicle, the tail region is composed of the posterior area including tail fin (posterior to the hindgut); the dorsal region is composed of the spinal cord, notochord, and somites (it begins directly posterior to the face region and ends directly anterior to the tail region), and the ventral region is composed of the entire gut area (it is directly inferior to the dorsal region, begins posterior to the face region and ends anterior to the tail region, before hindgut and anus). Since chromogenic ISH staining can contain some background enzyme activity, we standardized the cell counting and were able to differentiate one cell vs. a group of cells (red asterisk vs. white-dashed circle, respectively, in Supplementary Fig. S2d) using a custom ImageJ macro applied to images of embryos processed in the same ISH session. First, on clearly identifiable independent cells, we measured the smallest and biggest size (in pixels ${ }^{2}$ ) for one single positive cell. Then, we applied a threshold and adjusted the saturation to increase the contrast and segment cells from potential background. Finally, we indicated the minimal and maximal size for one independent cell on 'Analyze Particles' tool. The generated results were overlaid on the original image to remove possible artifacts. Number of positive cells per each region was normalized to the area in pixels ${ }^{2}$. The total number of cells per embryo was obtained as a sum of the four independent regions. Per each marker, spiba and $\mathrm{mmp7}$, inter- and intragroup comparisons were analyzed. Intergroup comparisons were performed among the different experimental groups (Ctrl, BR $\left.{ }^{-}, \mathrm{SC}^{-}, \mathrm{Tail}^{-}\right)$for each condition (NI vs. UTI) and per body region (face, tail, dorsal, ventral and total; Fig. 3c-l, Supplementary Fig. S2e-n). Intragroup comparisons were done between $\mathrm{NI}$ vs. UTI embryos, for each body region, within the same experimental group (Fig. 3e, o-r; Supplementary Fig. S2o-r). Particular care was taken to ensure that embryos from all the different groups per each condition were processed in the same batch at the same ISH session.

\section{Immunofluorescence and cell counting}

Cleaved Caspase-3 (Asp175; CC3), XL-2 (anti-Xenopus leukocytes, ${ }^{8}$ and acetylated alpha-tubulin (Tub) antibodies were used to detect apoptotic cells, ${ }^{43}$ leukocytes, ${ }^{8,43,83}$ and peripheral nerves, ${ }^{36,84}$ respectively, on wildtype and xlurp:::GFP embryos. Ctrl, $\mathrm{BR}^{-}, \mathrm{SC}^{-}$, Tail ${ }^{-}, \mathrm{NC}^{-}$and Simv-treated embryos were fixed $1 \mathrm{~h}$ in MEMFA ${ }^{76}$ and washed three times in PBS. They were then permeabilized in PBS-T for $30 \mathrm{~min}$ at room temperature (RT), followed by a $1 \mathrm{~h}$ blocking at room temperature in PBST supplemented with $10 \%$ heat-inactivated goat serum. The embryos were then incubated overnight at $4{ }^{\circ} \mathrm{C}$ with the primary antibody (monoclonal mouse anti-XL2 at 1:1000, kindly provided by Makoto Asashima's Lab at Tokyo University; polyclonal rabbit anti-CC3 at 1:300, Cell Signalling 9661; monoclonal mouse anti-Tub at 1:500, Sigma T7451). On the next day, they were washed six times in PBS ( $1 \mathrm{~h}$ each time, RT), before being incubated overnight with 
the secondary antibody (goat anti-mouse IgG conjugated with Alexa-Fluor 555 ; Invitrogen) at $4{ }^{\circ} \mathrm{C}$. The following day, animals were photographed using an Olympus BX-UCB microscope under $\times 4$ and $\times 10$ magnification, controlled by Metamorph software. Particular care was taken to ensure that embryos from all the different groups were processed in the same batch at the same immunofluorescence session.

The number of XL2- or CC3-positive cells was quantified and normalized to the tail area in pixels ${ }^{2}$ using ImageJ software. Leukocytes and apoptotic cells were counted along the $2 / 3$ posterior of the tail (Fig. 4a). To quantify number of GFP-positive cells (myeloid cells) and density of peripheral neural network in xlurp::GFP embryos, tails were subdivided in two independent regions: center and periphery (Fig. 4k, I). The somatic muscle or chevron-shaped myotomes composed 'center' region; 'peripheral' region was referred to the fin, both dorsal and ventral to the myotomes. Peripheral neural network was evaluated on images of stage 46-48 xlurp: GFP embryos that were immunoreacted against Tub, using gray-level measures (OD) as previously described. ${ }^{36,84-88}$ Two OD-mean values per animal were calculated after multiple measurements taken along the anteroposterior axis: one for center or somite region and one for peripheral fin. Analysis was done on raw black-and-white 8-bit images. Each measurement consisted of the mean value of the pixels of a fixed-size window. The size of the window remained constant across subjects. OD values ranging from 0 (black, no expression) to 255 (white, maximal expression). All individuals among whom comparisons are being made were produced in the same batch, treated identically for processing and imaging conditions were not changed. In addition, any potential aberrations originating from the optical system were corrected by background image subtraction.

\section{Next-generation sequencing}

Following infection and surgeries at st. 25 (brain removal, spinal cut, or tail amputation), embryos were grown for $3 \mathrm{~h}$ at $21^{\circ} \mathrm{C}$, then their anterior parts, including brain (to ensure that differences in RNA-Seq signature among samples were not coming from the brain tissue itself), were amputated and flash-frozen in groups of 20. RNA-Seq analyses were performed by The MIT BioMicro Center (Boston, MA). Samples were quality controlled on a Fragment Analyzer (Advanced Analytical) to determine DV200 scores. The mRNA from $1 \mu \mathrm{g}$ of total RNA was isolated using Illumina human/mouse/rat RiboZero Gold and prepared into Illumina libraries using the Kapa RNA HyperPrep kit and 10 cycles of amplification. Final libraries were pooled, and quality controlled using GPCR (Roche LC480II) as well as the Fragment Analyzer and sequenced on HiSeq2000.

Alignments were conducted by Genotypic Technology (India). Eight samples were aligned to the reference transcriptome Xenopus_laevis_v2, downloaded from NCBI [https://www.ncbi.nlm.nih.gov/genome/? term $=$ xenopus +laevis]. There were two biological replicates per treatment (brain intact, not infected or Ctrl Nl; brain intact infected or Ctrl UTI; brainless, not infected or $\mathrm{BR}^{-} \mathrm{Nl}$; brainless, infected or $\left.\mathrm{BR}^{-} \mathrm{UTI}\right)$. The raw data from Illumina were checked for quality using FastQC1 and preprocessed, which included removing the adapter sequences and removing the low-quality bases (<q30). Pre-processing of data was done with Cutadapt2. Mapping was performed with HISAT2 to align the high-quality data to the reference genome with the default parameters. Reads are classified into aligned reads (which align to the reference genome) and unaligned reads. HISAT2 is a fast and sensitive alignment program for mapping next-generation sequencing reads (both DNA and RNA) to a single reference genome). Based on an extension of BWT for graphs, ${ }^{89}$ a graph FM index (GFM) was implemented. HISAT2 uses a large set of small GFM indexes that collectively cover the whole genome. These small indexes (called local indexes), combined with several alignment strategies, enable rapid and accurate alignment of sequencing reads. This new indexing scheme is called a Hierarchical Graph FM index (HGFM).

Cufflinks was used to calculate transcript abundance. It results in normalized read count in the form of FPKM values. FPKM is a unit of measuring gene/transcript expression. Four comparisons were conducted (1) Ctrl NI vs. Ctrl UTI, (2) BR ${ }^{-} \mathrm{Nl}$ vs. BR ${ }^{-}$UTI, (3) Ctrl NI vs. BR ${ }^{-} \mathrm{Nl}$, and (4) Ctrl UTI vs. BR- UTI. Cufflinks includes a script called "Cuffmerge" that can be used to merge several Cufflinks assemblies together. The main purpose of this script is to develop an assembly GTF file suitable for use with Cuffdiff. Merged GTF files produced by Cuffmerge were used as input in Cuffdiff. Cuffdiff was used to calculate the differentially expressed transcripts and generated $p$-values, FDR corrected $p$-values ( $q$ value) and the $\log _{2}$ fold change values. RNA-Seq unprocessed and processed data have been deposited in the National Center for Biotechnology Information (NCBI)
Gene Expression Omnibus (GEO) and is accessible through GEO Series accession number GSE119729. Supplementary Data 1 contains all processed transcript data, including fold change, $p$-value, and $q$-value.

Sub-network enrichment analysis (SNEA) was used to identify networks enriched in each of the four comparisons. Analyses were conducted in Pathway Studio 10.0 (Elsevier) using the ResNet 11.0. The number of gene symbols that mapped to the mammalian homologs in the program ranged from $\sim 12,300$ to 12,500 for each of the four datasets using the official gene Name + Alias. Duplicates were addressed using the default "best $p$-value if present, or maximum fold change" in Pathway Studio. Sub-networks related to cell process were queried and there were 1000 permutations of the data using Kolmogorov-Smirnov algorithm to generate the distributions. SNEA identifies gene networks related to cellular processes or diseases that change with a treatment or disease; these networks are predefined molecular networks (expression patterns, binding, or involvement in common pathways) based upon the literature that are focused around gene hubs. All transcripts identified by RNA-seq were used in the analysis and acted as the background list for enrichment. See Supplementary Methods 1 for Reads and quality of sequencing.

\section{Dopamine functional assays}

Liquid chromatography-mass spectrometry (LC-MS/MS): UTI-infected Ctrl and $\mathrm{BR}^{-}$embryos were harvested at early st. $35(\sim 20 \mathrm{~h}$ post-surgery, $44 \mathrm{~h}$ post infection) immediately before the peak of infection-induced death in $\mathrm{BR}^{-}$animals and the significant differences both in survival rate and apoptosis are reached respect to Ctrl embryos (experimentally defined in advance; see Fig. 2a, b). Three biological replicates per experimental group were analyzed for DA level quantification. Each replicate consisted of 30 embryos. Their anterior parts, including brain, were amputated to ensure that differences in DA levels among samples were not coming from the brain tissue itself, and then flash-frozen in groups of 30 embryos. LC-MS/ MS was performed by The Small Molecule Mass Spectrometry in the Faculty of Arts and Sciences at Harvard University (Cambridge, MA). LC-MS grade dopamine hydrochloride (DA) and Dopamine-1,1,2,2-d4 hydrochloride (d4) were purchased from Millipore Sigma (cat. D-081, 73483). The working standard $(\mathrm{DA}, 0.61 \mathrm{ng} / \mathrm{mL})$ and working internal standard solutions (DA-d4, $50 \mathrm{ng} / \mathrm{mL}$ ) were diluted in LC-MS grade water. Sample preparation and protein precipitation were performed by adding $50 \mu \mathrm{L}$ of $100 \mathrm{pg} / \mu \mathrm{L}$ of DA-d4 and $200 \mu \mathrm{L}$ of cold methanol. The LC-MS/MS analysis was done on an Agilent 6460 Triple-quad mass spectrometer (Agilent Technologies) coupled to an Agilent 1290 uHPLC. The chromatographic separation was performed using an Agilent Eclipse XDB ( $2.1 \mathrm{~mm}$ internal diameter $\times 50 \mathrm{~mm}$ length $\times 2.7 \mu \mathrm{m}$ particle size) $\mathrm{C} 18$ column. A constant flow rate of $0.400 \mathrm{~L} / \mathrm{min}$ was used, along with a $10 \mu \mathrm{L}$ injection volume. Mobile phase $A$ was $0.1 \%$ formic acid in water $v / v$ and $B$ was $0.1 \%$ formic acid in acetonitrile $v / v$. Initial conditions were $98 \%$ A, 2\% B. Compounds were eluted by increasing mobile phase composition to $98 \%$ B over 5 min, and the columns re-equilibrated to starting conditions for $3 \mathrm{~min}$ prior to the next injection. Targeted analysis using multiple reaction monitoring (MRM) monitoring two transitions for DA and DA-d4 using the following transitions: DA: $154 \rightarrow 137$ and $154 \rightarrow 91$, and DA-d4: $\mathrm{m} / \mathrm{z} \quad 158 \rightarrow 141$ and $158 \rightarrow 95$. The optimized collision energy was 9 and $25 \mathrm{~V}$, respectively, for the first and second transition. A fragmentor value of $90 \mathrm{~V}$ was employed. The standard curve was measured between $0.1 \mathrm{pg} / \mu \mathrm{L}$ and $50 \mathrm{pg}$ in water. The quantitative analysis was performed in MassHunter Quantitative Analysis software (Agilent Technologies, Santa Clara, USA). Data were collected from compounds with a $\mathrm{S} / \mathrm{N}$ ratio of $>10$. A linear curve fit with a $1 / x$ weighting was used. See Supplementary Methods 2 for LC-MS/MS conditions and parameters.

Drug Exposure. Uninfected and UTI-infected Ctrl and $\mathrm{BR}^{-}$Xenopus embryos were exposed to specific pharmacological agents, targeting the type-1 or type-2 family of dopamine (DA) receptors (D1R, D2R), from st. 25 (immediately after brain removal) to st. 48. The drugs were refreshed every day. We used specific agonists and antagonists of each dopamine receptor: $10 \mu$ M SKF-38393, D1R agonist (SKF, Tocris 0922); $10 \mu \mathrm{M} \mathrm{SCH-23390,} \mathrm{D1R}$ antagonist ( $\mathrm{SCH}$, Tocris 0925); $10 \mu \mathrm{M}$ Quinpirole, D2R agonist (Quin, Tocris 1061); $1 \mu \mathrm{M} \mathrm{L-741,626,} \mathrm{D2R} \mathrm{antagonist} \mathrm{(L741,} \mathrm{Tocris} \mathrm{1003).} \mathrm{All} \mathrm{drug}$ treatments were performed using embryos from mixed batches of fertilizations and using three biological replicates per drug. Each replicate consisted of 30 embryos. Stock solutions of SKF, SCH and Quin were created by dissolving the compound in Millipore water to a final drug concentration of $100 \mathrm{mM}$ for both SCH and Quin, and to $25 \mathrm{mM}$ for SKF. Stock solution of L741 was created by dissolving the compound in DMSO to a final drug concentration of $100 \mathrm{mM}$. All aliquots were stored at $-20^{\circ} \mathrm{C}$. 
Further dilution of all compounds was made in $20 \mathrm{ml}$ of normal frog media (0.1X MMR). Control experiments were performed using embryos in $0.1 \mathrm{X}$ MMR (no drug), both for Ctrl and $\mathrm{BR}^{-}$groups. Drug concentrations were determined through toxicity screens and were applied at levels that did not result in lethality or observable developmental defects.

\section{Statistics}

All statistical analysis was performed using GraphPad Prism (GraphPad Software, Inc., CA, USA). Animal numbers were chosen based on previous studies as well as sample size estimates given a type I error rate of $5 \%$ and power of 0.8. Each dish of tadpoles was considered a replicate. After applying Bartlett's test (for equal variances), data from various replicates and multiple independent groups were analyzed by one-way or two-way ANOVA test (if equal variances) or Kruskal-Wallis test (if significantly different variances). When $P<0.05$, post hoc multiple comparisons were performed by Bonferroni or Dunn test, respectively. The significance level (a) was set to 0.05 in all cases. $P$ value on graphs is after post hoc Bonferroni or Dunn analysis. The statistical values are reported as mean \pm SD. Number of replicates $(r)$, number of animals per replicate $(n)$, number of total animals $(N=r \times n)$, and specific analysis used for each experiment are stated in the "Results" section and/or figure legends.

\section{Reporting summary}

Further information on research design is available in the Nature Research Reporting Summary linked to this article.

\section{DATA AVAILABILITY}

The authors declare that all data supporting the findings of this study are available within the article and its Supplementary Information Files or from the corresponding author upon reasonable request. RNA-seq data have been deposited into the NCBI Gene Expression Omnibus (GEO) database (Accession number: GSE119729).

Received: 4 June 2019; Accepted: 20 December 2019; Published online: 04 February 2020

\section{REFERENCES}

1. Robert, J. \& Ohta, Y. Comparative and developmental study of the immune system in Xenopus. Dev. Dyn. 238, 1249-1270 (2009).

2. Kim, M. S. et al. Rapid linkage of innate immunological signals to adaptive immunity by the brain-fat axis. Nat. Immunol. 16, 525-533 (2015).

3. Agricola, Z. N. et al. Identification of genes expressed in the migrating primitive myeloid lineage of Xenopus laevis. Dev. Dyn. 245, 47-55 (2016).

4. Du Pasquier, L., Schwager, J. \& Flajnik, M. F. The immune system of Xenopus. Annu. Rev. Immunol. 7, 251-275 (1989).

5. Paredes, R., Ishibashi, S., Borrill, R., Robert, J. \& Amaya, E. Xenopus: an in vivo model for imaging the inflammatory response following injury and bacterial infection. Dev. Biol. 408, 213-228 (2015).

6. Robert, J., Gantress, J., Cohen, N. \& Maniero, G. D. Xenopus as an experimental model for studying evolution of hsp-immune system interactions. Methods 32, 42-53 (2004).

7. Ciau-Uitz, A. \& Liu, F. \& Patient, R. Genetic control of hematopoietic development in Xenopus and zebrafish. Int J. Dev. Biol. 54, 1139-1149 (2010).

8. Miyanaga, Y., Shiurba, R., Nagata, S., Pfeiffer, C. J. \& Asashima, M. Induction of blood cells in Xenopus embryo explants. Dev. Genes Evolution 207, 417-426 (1998).

9. Pare, J. F., Martyniuk, C. J. \& Levin, M. Bioelectric regulation of innate immune system function in regenerating and intact Xenopus laevis. NPJ Regenerative Med. 2, 15 (2017).

10. Costa, R. M., Soto, X., Chen, Y., Zorn, A. M. \& Amaya, E. spib is required for primitive myeloid development in Xenopus. Blood 112, 2287-2296 (2008)

11. Tomlinson, M. L., Garcia-Morales, C., Abu-Elmagd, M. \& Wheeler, G. N. Three matrix metalloproteinases are required in vivo for macrophage migration during embryonic development. Mechanisms Dev. 125, 1059-1070 (2008).

12. Filiano, A. J., Gadani, S. P. \& Kipnis, J. Interactions of innate and adaptive immunity in brain development and function. Brain Res. 1617, 18-27 (2015).

13. Filiano, A. J., Gadani, S. P. \& Kipnis, J. How and why do T cells and their derived cytokines affect the injured and healthy brain? Nat. Rev. Neurosci. 18, 375-384 (2017).
14. Rosenberg, M. D. Baby brains reflect maternal inflammation. Nat. Neurosci. 21, 651-653 (2018).

15. Filiano, A. J. et al. Unexpected role of interferon-gamma in regulating neuronal connectivity and social behaviour. Nature 535, 425-429 (2016).

16. Kipnis, J., Gadani, S. \& Derecki, N. C. Pro-cognitive properties of T cells. Nat. Rev. Immunol. 12, 663-669 (2012).

17. Louveau, A. et al. Structural and functional features of central nervous system lymphatic vessels. Nature 523, 337-341 (2015).

18. Kipnis, J. \& Filiano, A. J. Neuroimmunology in 2017: the central nervous system: privileged by immune connections. Nat. Rev. Immunol. 18, 83-84 (2018).

19. Louveau, A., Harris, T. H. \& Kipnis, J. Revisiting the mechanisms of CNS immune privilege. Trends Immunol. 36, 569-577 (2015).

20. Elenkov, I. J., Wilder, R. L., Chrousos, G. P. \& Vizi, E. S. The sympathetic nerve-an integrative interface between two supersystems: the brain and the immune system. Pharmacol. Rev. 52, 595-638 (2000).

21. Vasamsetti, S. B. et al. Sympathetic neuronal activation triggers myeloid progenitor proliferation and differentiation. Immunity 49, 93-106.e107 (2018).

22. Kipnis, J. Immune system: the "seventh sense". J. Exp. Med. 215, 397-398 (2018).

23. Styer, K. L. et al. Innate immunity in Caenorhabditis elegans is regulated by neurons expressing NPR-1/GPCR. Science 322, 460-464 (2008).

24. Ewbank, J. J. \& Pujol, N. Local and long-range activation of innate immunity by infection and damage in C. elegans. Curr. Opin. Immunol. 38, 1-7 (2016).

25. Meisel, J. D., Panda, O., Mahanti, P., Schroeder, F. C. \& Kim, D. H. Chemosensation of bacterial secondary metabolites modulates neuroendocrine signaling and behavior of C. elegans. Cell 159, 267-280 (2014).

26. Gadani, S. P. \& Kipnis, J. Brainless immunity no more. Nat. Immunol. 16, 440-441 (2015).

27. Tandon, P., Conlon, F., Furlow, J. D. \& Horb, M. E. Expanding the genetic toolkit in Xenopus: Approaches and opportunities for human disease modeling. Dev. Biol. 426, 325-335 (2017).

28. Maia, L. A., Velloso, I. \& Abreu, J. G. Advances in the use of Xenopus for successful drug screening. Expert Opin. Drug Discov. 1-7. https://doi.org/10.1080/ 17460441.2017.1367281 (2017)

29. Getwan, M. \& Lienkamp, S. S. Toolbox in a tadpole: Xenopus for kidney research. Cell Tissue Res. https://doi.org/10.1007/s00441-017-2611-2 (2017).

30. Dubey, A. \& Saint-Jeannet, J. P. Modeling human craniofacial disorders in Xenopus. Curr. Pathobiol. Rep. 5, 79-92 (2017).

31. Banach, M. \& Robert, J. Tumor immunology viewed from alternative animal models-the Xenopus story. Curr. Pathobiol. Rep. 5, 49-56 (2017).

32. Duncan, A. R. \& Khokha, M. K. Xenopus as a model organism for birth defectsCongenital heart disease and heterotaxy. Semin Cell Dev. Biol. 51, 73-79 (2016).

33. Hardwick, L. J. \& Philpott, A. An oncologists friend: how Xenopus contributes to cancer research. Dev. Biol. 408, 180-187 (2015).

34. Schmitt, S. M., Gull, M. \& Brandli, A. W. Engineering Xenopus embryos for phenotypic drug discovery screening. Adv. Drug Deliv. Rev. 69-70, 225-246 (2014).

35. Herrera-Rincon, C. \& Levin, M. Booting up the organism during development: Prebehavioral functions of the vertebrate brain in guiding body morphogenesis. Commun. Integr. Biol. 11, e1433440 (2018).

36. Herrera-Rincon, C., Pai, V. P., Moran, K. M., Lemire, J. M. \& Levin, M. The brain is required for normal muscle and nerve patterning during early Xenopus development. Nat. Commun. 8, 587 (2017).

37. Huang, C. \& Niethammer, P. Tissue damage signaling is a prerequisite for protective neutrophil recruitment to microbial infection in zebrafish. Immunity 48, 1006-1013.e1006 (2018).

38. Thompson, P. D., Clarkson, P. \& Karas, R. H. Statin-associated myopathy. Jama 289, 1681-1690 (2003).

39. Campos, L. M. et al. Alterations in zebrafish development induced by simvastatin: comprehensive morphological and physiological study, focusing on muscle. Exp. Biol. Med. 241, 1950-1960 (2016).

40. Campos, L. M. et al. Structural analysis of alterations in zebrafish muscle differentiation induced by simvastatin and their recovery with cholesterol. J. Histochem. Cytochem. 63, 427-437 (2015).

41. Ayres, J. S. \& Schneider, D. S. Tolerance of infections. Annu. Rev. Immunol. 30, 271-294 (2012).

42. Soares, M. P., Teixeira, L. \& Moita, L. F. Disease tolerance and immunity in host protection against infection. Nat. Rev. Immunol. 17, 83-96 (2017).

43. Tseng, A.-S., Adams, D. S., Qiu, D., Koustubhan, P. \& Levin, M. Apoptosis is required during early stages of tail regeneration in Xenopus laevis. Developmental Biol. 301, 62-69 (2007).

44. Makino, S. et al. Heat-shock protein 60 is required for blastema formation and maintenance during regeneration. Proc. Natl. Acad. Sci. USA 102, 14599-14604 (2005).

45. Crowley, L. C. \& Waterhouse, N. J. Detecting cleaved caspase-3 in apoptotic cells by flow cytometry. Cold Spring Harb. 2016. https://doi.org/10.1101/pdb. prot087312 (2016). 
46. Lichanska, A. M. et al. Differentiation of the mononuclear phagocyte system during mouse embryogenesis: the role of transcription factor PU.1. Blood 94, 127-138 (1999).

47. Su, G. H. et al. Defective B cell receptor-mediated responses in mice lacking the Ets protein, Spi-B. EMBO J. 16, 7118-7129 (1997).

48. Harrison, M. et al. Matrix metalloproteinase genes in Xenopus development. Dev. Dyn. 231, 214-220 (2004).

49. Grayfer, L. \& Robert, J. Amphibian macrophage development and antiviral defenses. Developmental Comp. Immunol. 58, 60-67 (2016).

50. Hansen, J. D. \& Zapata, A. G. Lymphocyte development in fish and amphibians. Immunological Rev. 166, 199-220 (1998).

51. Imai, Y. et al. Multiple origins of embryonic and tadpole myeloid cells in Xenopus laevis. Cell Tissue Res. 369, 341-352 (2017).

52. Smith, S. J., Kotecha, S., Towers, N., Latinkic, B. V. \& Mohun, T. J. XPOX2-peroxidase expression and the XLURP-1 promoter reveal the site of embryonic myeloid cell development in Xenopus. Mechanisms Dev. 117, 173-186 (2002).

53. Chang, J., Baker, J. \& Wills, A. Transcriptional dynamics of tail regeneration in Xenopus tropicalis. Genesis 55. https://doi.org/10.1002/dvg.23015 (2017).

54. Hutchins, E. D. et al. Transcriptomic analysis of tail regeneration in the lizard Anolis carolinensis reveals activation of conserved vertebrate developmental and repair mechanisms. PLoS ONE 9, e105004 (2014)

55. McLaughlin, K. A. \& Levin, M. Bioelectric signaling in regeneration: Mechanisms of ionic controls of growth and form. Dev. Biol. 433, 177-189 (2018).

56. Mathews, J. \& Levin, M. The body electric 2.0: recent advances in developmental bioelectricity for regenerative and synthetic bioengineering. Curr. Opin. Biotechnol. 52, 134-144 (2018).

57. Kipnis, J. et al. Dopamine, through the extracellular signal-regulated kinase pathway, downregulates CD4+CD25+ regulatory T-cell activity: implications for neurodegeneration. J. Neurosci. 24, 6133-6143 (2004).

58. Levite, M. Dopamine and T cells: dopamine receptors and potent effects on $\mathrm{T}$ cells, dopamine production in T cells, and abnormalities in the dopaminergic system in T cells in autoimmune, neurological and psychiatric diseases. Acta Physiologica 216, 42-89 (2016).

59. Jia, J.-M., Zhao, J., Hu, Z., Lindberg, D. \& Li, Z. Age-dependent regulation of synaptic connections by dopamine D2 receptors. Nat. Neurosci. 16, 1627 (2013).

60. Prado, C. et al. Stimulation of dopamine receptor D5 expressed on dendritic cells potentiates Th17-mediated immunity. J. Immunol. 188, 3062-3070 (2012).

61. Mori, T. et al. D1-like dopamine receptors antagonist inhibits cutaneous immune reactions mediated by Th2 and mast cells. J. Dermatological Sci. 71, 37-44 (2013).

62. Picton, L. D. \& Sillar, K. T. Mechanisms underlying the endogenous dopaminergic inhibition of spinal locomotor circuit function in Xenopus tadpoles. Sci. Rep. 6, 35749 (2016).

63. Pavlov, V. A., Chavan, S. S. \& Tracey, K. J. Molecular and functional neuroscience in immunity. Annu. Rev. Immunol. 36, 783-812 (2018).

64. Kipnis, J. Multifaceted interactions between adaptive immunity and the central nervous system. Science 353, 766-771 (2016).

65. Fukazawa, T., Naora, Y., Kunieda, T. \& Kubo, T. Suppression of the immune response potentiates tadpole tail regeneration during the refractory period. Development 136, 2323-2327 (2009).

66. Smith, S. N., Hagan, E. C., Lane, M. C. \& Mobley, H. L. T. Dissemination and systemic colonization of uropathogenic Escherichia coli in a murine model of bacteremia. MBio 1, e00262-10 (2010).

67. Wang, M.-I et al. A rabbit model of right-sided Staphylococcus aureus endocarditis created with echocardiographic guidance. Cardiovasc. Ultrasound 11, 3-3 (2013).

68. Cantral, D. E. et al. Quantitative culture of bronchoalveolar lavage fluid for the diagnosis of bacterial pneumonia. Am. J. Med. 95, 601-607 (1993).

69. Bradley, J. M. \& Little, P. J. Quantitative urine cultures. Br. Med. J. 2, 361-363 (1963).

70. Yaparla, A., Wendel, E. S. \& Grayfer, L. The unique myelopoiesis strategy of the amphibian Xenopus laevis. Developmental Comp. Immunol. 63, 136-143 (2016).

71. Micheau, O. \& Tschopp, J. Induction of TNF receptor I-mediated apoptosis via two sequential signaling complexes. Cell 114, 181-190 (2003).

72. Nish, S. \& Medzhitov, R. Host defense pathways: role of redundancy and compensation in infectious disease phenotypes. Immunity 34, 629-636 (2011).

73. Zhang, X., Liu, Q., Liao, Q. \& Zhao, Y. Potential roles of peripheral dopamine in tumor immunity. J. Cancer 8, 2966-2973 (2017).

74. Talhada, D., Rabenstein, M. \& Ruscher, K. The role of dopaminergic immune cell signalling in poststroke inflammation. Ther. Adv. Neurol. Diso. 11 1756286418774225 (2018).

75. Jiang, Y., Woronicz, J. D., Liu, W. \& Goeddel, D. V. Prevention of constitutive TNF receptor 1 signaling by silencer of death domains. Science 283, 543-546 (1999).

76. Sive, H. L., Grainger, R. M. \& Harland, R. M. Early Development of Xenopus Laevis: A Laboratory Manual (Cold Spring Harbor Laboratory Press, 2000).
77. Pearl, E. J., Grainger, R. M., Guille, M. \& Horb, M. E. Development of Xenopus resource centers: the National Xenopus Resource and the European Xenopus Resource Center. Genesis 50, 155-163 (2012).

78. Nieuwkoop, P. D. \& Faber, J. Normal Table of Xenopus Laevis (Daudin): A Systematical and Chronological Survey of the Development from the Fertilized Egg Till the End of Metamorphosis. (Garland Pub., 1994).

79. Wiles, T. J., Bower, J. M., Redd, M. J. \& Mulvey, M. A. Use of zebrafish to probe the divergent virulence potentials and toxin requirements of extraintestinal pathogenic Escherichia coli. PLoS Pathog. 5, e1000697 (2009).

80. Lee, Y. H. \& Saint-Jeannet, J. P. Cardiac neural crest is dispensable for outflow tract septation in Xenopus. Development 138, 2025-2034 (2011).

81. Dontje, W. et al. Delta-like1-induced Notch1 signaling regulates the human plasmacytoid dendritic cell versus T-cell lineage decision through control of GATA-3 and Spi-B. Blood 107, 2446-2452 (2006)

82. Li, Q., Park, P. W., Wilson, C. L. \& Parks, W. C. Matrilysin shedding of syndecan-1 regulates chemokine mobilization and transepithelial efflux of neutrophils in acute lung injury. Cell 111, 635-646 (2002).

83. Chernet, B. T. \& Levin, M. Transmembrane voltage potential is an essential cellular parameter for the detection and control of tumor development in a Xenopus model. Dis. Model Mech. 6, 595-607 (2013).

84. Chu, D. T. \& Klymkowsky, M. W. The appearance of acetylated alpha-tubulin during early development and cellular differentiation in Xenopus. Dev. Biol. 136, 104-117 (1989).

85. Slaughter, T., Wang, J. \& Black, M. M. Microtubule transport from the cell body into the axons of growing neurons. J. Neurosci. 17, 5807-5819 (1997).

86. Seyed Jafari, S. M. \& Hunger, R. E. IHC optical density score: a new practical method for quantitative immunohistochemistry image analysis. Appl. Immunohisto. Mol. Morphol. 25, e12-e13 (2017).

87. Seyed Jafari, S. M. et al. Correlation of Vascular Endothelial Growth Factor subtypes and their receptors with melanoma progression: a next-generation Tissue Microarray (ngTMA) automated analysis. PLoS ONE 13, e0207019 (2018).

88. Rizzardi, A. E. et al. Quantitative comparison of immunohistochemical staining measured by digital image analysis versus pathologist visual scoring. Diagnostic Pathol. 7, 42 (2012)

89. Sir, J. et al. Indexing graphs for path queries with applications in genome research. IEEE/ACM Trans. Comput. Biol. Bioinforma. 11, 375-388 (2014).

\section{ACKNOWLEDGEMENTS}

We thank Erin Switzer and Rakela Colon for Xenopus husbandry and general laboratory assistance; Matthew M. Mulvey (University of Utah) for providing the E. coli strains; Enrique Amaya (University of Manchester) for providing the spiba and $\mathrm{mmp}$ 7 probes; Joan Lemire for assistance with molecular biology; Vikas Trivedi and Todd Chappell for assistance with E. coli preparation; Kelly McLaughlin and Joshua Finkelstein for their helpful comments on the paper; Kelly Chatman and Sunia Truger for performing the LC-MS/MS experiments. XL-2 antibody was a generous gift from Makoto Asashima's laboratory at Tokyo University. The methods were performed in "accordance" with relevant guidelines and regulations. We gratefully acknowledge support of the Templeton World Charity Foundation Independent Research Fellowship to C.H.-R. (TWCF0241) and the Allen Discovery Center program through The Paul G. Allen Frontiers Group (12171), as well as DARPA (W911NF-16-C-0050), and the NIH (AR055993, AR061988).

\section{AUTHOR CONTRIBUTIONS}

C.H.-R. and J.-F.P. performed experiments. C.J.M. analyzed RNA-seq data and prepared several figures and tables. M.L., C.H.-R. and J.-F.P. designed the experiments and interpreted data. S.K.J., C.H., and A.F. assisted with the immunofluorescence experiments and cell counting. V.K. and A.D. performed bacterial injection and analysis. C.H.-R., C.-J.M., J.-F.P. and M.L. wrote the paper together.

\section{COMPETING INTERESTS}

The authors declare no competing interests.

\section{ADDITIONAL INFORMATION}

Supplementary information is available for this paper at https://doi.org/10.1038/ s41536-020-0087-2.

Correspondence and requests for materials should be addressed to M.L. 
Reprints and permission information is available at http://www.nature.com/ reprints

Publisher's note Springer Nature remains neutral with regard to jurisdictional claims in published maps and institutional affiliations.

cc (i) Open Access This article is licensed under a Creative Commons Attribution 4.0 International License, which permits use, sharing, adaptation, distribution and reproduction in any medium or format, as long as you give appropriate credit to the original author(s) and the source, provide a link to the Creative Commons license, and indicate if changes were made. The images or other third party material in this article are included in the article's Creative Commons license, unless indicated otherwise in a credit line to the material. If material is not included in the article's Creative Commons license and your intended use is not permitted by statutory regulation or exceeds the permitted use, you will need to obtain permission directly from the copyright holder. To view a copy of this license, visit http://creativecommons. org/licenses/by/4.0/.

(c) The Author(s) 2020 